\title{
Active Control of Wind Turbines Through Varying Blade Tip Sweep
}

\author{
Boulamatsis, Achilles M.; Barlas, Thanasis K.; Stapountzis, Herricos
}

Published in:

Renewable Energy

Link to article, DOI:

10.1016/j.renene.2018.07.022

Publication date:

2019

Document Version

Peer reviewed version

Link back to DTU Orbit

Citation (APA):

Boulamatsis, A. M., Barlas, T. K., \& Stapountzis, H. (2019). Active Control of Wind Turbines Through Varying Blade Tip Sweep. Renewable Energy, 131, 25-36. https://doi.org/10.1016/j.renene.2018.07.022

\section{General rights}

Copyright and moral rights for the publications made accessible in the public portal are retained by the authors and/or other copyright owners and it is a condition of accessing publications that users recognise and abide by the legal requirements associated with these rights.

- Users may download and print one copy of any publication from the public portal for the purpose of private study or research.

- You may not further distribute the material or use it for any profit-making activity or commercial gain

- You may freely distribute the URL identifying the publication in the public portal

If you believe that this document breaches copyright please contact us providing details, and we will remove access to the work immediately and investigate your claim. 


\section{Accepted Manuscript}

Active Control of Wind Turbines Through Varying Blade Tip Sweep

Achilles M. Boulamatsis, Thanasis K. Barlas, Herricos Stapountzis

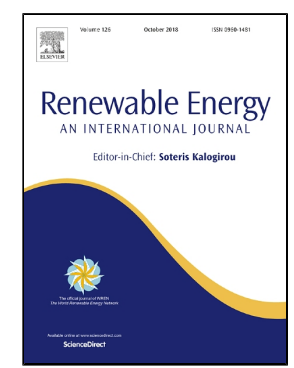

PII:

S0960-1481(18)30819-X

DOI:

10.1016/j.renene.2018.07.022

Reference:

RENE 10296

To appear in:

Renewable Energy

Received Date:

20 November 2017

Accepted Date:

05 July 2018

Please cite this article as: Achilles M. Boulamatsis, Thanasis K. Barlas, Herricos Stapountzis, Active Control of Wind Turbines Through Varying Blade Tip Sweep, Renewable Energy (2018), doi: 10.1016/j.renene.2018.07.022

This is a PDF file of an unedited manuscript that has been accepted for publication. As a service to our customers we are providing this early version of the manuscript. The manuscript will undergo copyediting, typesetting, and review of the resulting proof before it is published in its final form. Please note that during the production process errors may be discovered which could affect the content, and all legal disclaimers that apply to the journal pertain. 


\title{
Active Control of Wind Turbines Through Varying Blade Tip Sweep
}

\author{
Achilles M. Boulamatsis*, Thanasis K. Barlas*+, Herricos Stapountzis* \\ Laboratory of Fluid Mechanics and Turbomachinery, University Of Thessaly Pedion Areos Volos \\ Greece* \\ Aerodynamic Design Section, Department of Wind Energy, Technical University of Denmark (DTU) ${ }^{+}$ \\ aboulamatsis@mie.uth.gr
}

\begin{abstract}
In this research work an introduction to the concept of an actively controlled horizontal axis wind turbine through varying blade tip sweep, is presented. The concept refers to variable tip swept rotor blades, that have the ability to pivot collectively aft, about an axis located at the blade tips. Quantities to be controlled are power production and blade loads. The investigation is carried out with a modified Blade Element Momentum (BEM) model that takes into account variable tip swept rotor blades and the modifications are based on results from a lifting line theory based model. The simulations refer to the 5MW NREL reference wind turbine that incorporates a suitable controller and preliminary results show beneficial behaviour in all of the investigated areas.
\end{abstract}

Keywords - Active Control, Swept Blades, Unsteady Lifting Line Theory, Blade Element Momentum Theory

Abbreviations:

AEP: Annual Energy Production

AOA: Angle Of Attack

BEM: Blade Element Momentum (Theory)

CFD: Computational Fluid Dynamics

CUDA: Compute Unified Device Architecture

DEL: Damage Equivalent Load

DU_SWAMP: Delft University Smart Wind turbine Aeroelastic Modular Processing (model)

$\mathbf{E C}$ : Energy research Centre of the Netherlands

EOG: Extreme Operating Gust

IEC: International Electrotechnical Commission

MW: Megawatt

NREL: National Renewable Energy Laboratory

STAR: Swept Twist Adaptive Rotor

TE: Trailing Edge

TurbSim: Turbulence Simulator

ULL: Unsteady Lifting Line (Theory)

\section{List of Symbols:}

A: cross section area - rotor swept area

$\mathbf{A}_{\text {mp(x)s }}$ : amplitude of a wind turbine parameter due to the harmonic sweeping motion of the blade tip

aif: axial induction factor

$\mathbf{C}_{\mathbf{L}}$ : Lift coefficient

$\mathbf{C}_{\text {LSw }}$ : Lift coefficient of a swept wing

$\mathbf{C}_{\mathbf{P}}$ : power coefficient

Circ $_{\text {diff }}$ : bound circulation difference between adjacent blade elements 
c: chord length of a blade or a blade section

F: external force

$\mathbf{f}_{\mathrm{sw}}$ :frequency of the sweeping motion of the blade tip

$\mathbf{f}_{\text {rot }}$ :rotational frequency of the rotor

f: frequency of motion

G: correction factor (for blade tip sweep)

$\mathrm{g}_{1}$ : correction factor 1 (for blade tip sweep)

$\mathbf{g}_{2}$ : correction factor 2 (for blade tip sweep)

$\mathbf{K}$ : controller gain

I: length of a vortex filament

Mean $_{\mathbf{M x}}$ : average of blade root bending moments

$\mathbf{M}_{\mathbf{x}}$ : blade root bending moment

$\mathbf{N}_{\text {ratio }}$ : non-dimensional variable - amplitude of parameter divided by the same parameter value in stable conditions

P: power

$\mathbf{R}$ : rotor diameter

$\mathbf{r}$ : distance of a point from a vortex segment - distance of a section from the rotor hub center

T: thrust force

t: time

V: Wind velocity

$\mathbf{V}_{\text {ind }}$ : induced velocity on a single point

$\mathbf{V}_{\text {inflow: }}$ : wind turbine inflow velocity

$\mathbf{W}$ : wake velocity

$\mathbf{X}_{\mathbf{C p G}}$ : vertical distance travelled in-plane by blade elements according to blade tip sweep

$\mathbf{X}_{\mathbf{0}}$ : Parameter value in stable operating conditions

$\mathbf{y}$ : distance in y direction

$\boldsymbol{\alpha}:$ axial induction factor

$\boldsymbol{\alpha}^{\prime}$ : tangential induction factor

$\delta$ r: percentage of vortex filament length

$\Gamma$ : blade circulation - vortex strength

$\Lambda$ : sweep angle

$\Lambda_{1}$ : longitudinal turbulence scale parameter

р: air density

$\boldsymbol{\varphi}$ : inflow angle

$\omega:$ angular velocity of the rotor

\section{Subscripts:}

sw: swept

\section{INTRODUCTION}

Over the past few years there is a continuous effort for increasing energy production and reducing dynamic loads of wind turbines. In [1] there is an extensive review of the current status in smart rotor control that goes beyond the borders of conventional control methods like pitch or stall regulation. In particular, smart rotor control refers to an integrated system equipped with sensors, actuators and one or more microprocessors that operate in a feedback loop and control the blade aerodynamic loads. The latter is achieved either by enhancing the flow around the blade with the deployment of microtabs and use of boundary layer control methods (like vortex generators and active synthetic jets) or by altering the shape of the airfoil utilizing camber control, active twist or flaps. However, all of these features have to be carefully designed in order to compensate for their complexity and the fault cases that they may impose.

In this research work an introduction to an innovative control method is presented through tip swept rotor blades that have the ability to pivot simultaneously aft (in-plane movement) about an axis located at the blade tips. The swept tip can be either part of the main blade with an internal mechanism or an added 
surface (add-on) to the blades, as it is shown in Figure 1. The purpose of this control feature is to actively adjust power at specific operating areas and reduce fatigue blade loads or extreme loads during a wind gust through small sweep angle variations in the tip area. Similar research efforts like [2], [3] and [4] have already concluded that aft sweeping of blades plays a beneficial role on reducing the loads while fore sweeping increases them. However, the aforementioned methods refer to passive load control methods, in which changes in wind speed are counteracted through the passively adapting aeroelastic response of the rotor blades (for example tension - torsion, bend - twist, sweep - twist coupling), whereas this research intends to fill in the gap of active load/power control through varying blade tip sweep for an otherwise nondeformable rotor.

Apart from the well known CFD methods that can be used to examine the impact of blade geometry modifications on wind turbine aerodynamics other tools are Lifting Line theory and Blade Element Momentum (BEM) theory. With lifting line theory geometry features like tip sweep are efficiently modeled in applications where high aspect ratio wings are involved. Aerodynamic lift is modeled through vortex distribution over the blades which then creates a vortex sheet behind the turbine. Sweep angle changes have a direct effect on this distribution and of course on the overall aerodynamics. However, some configurations of the vortex method can elevate the computational cost as high as CFD and so it not always a straightforward choice. BEM method has always been an attractive choice for wind turbine applications because of its relative simplicity, yet it has not been used with variable sweep angle applications due to its fundamental assumption for no radial flow interaction.

Sweep effect on lift is expressed through the simple cosine law (presented later with equation 8) when a fixed wing is considered but the rotation of the wind turbine blades combined with the rotation of a small part of the blade introduces bigger challenges to the modeling of the whole concept.

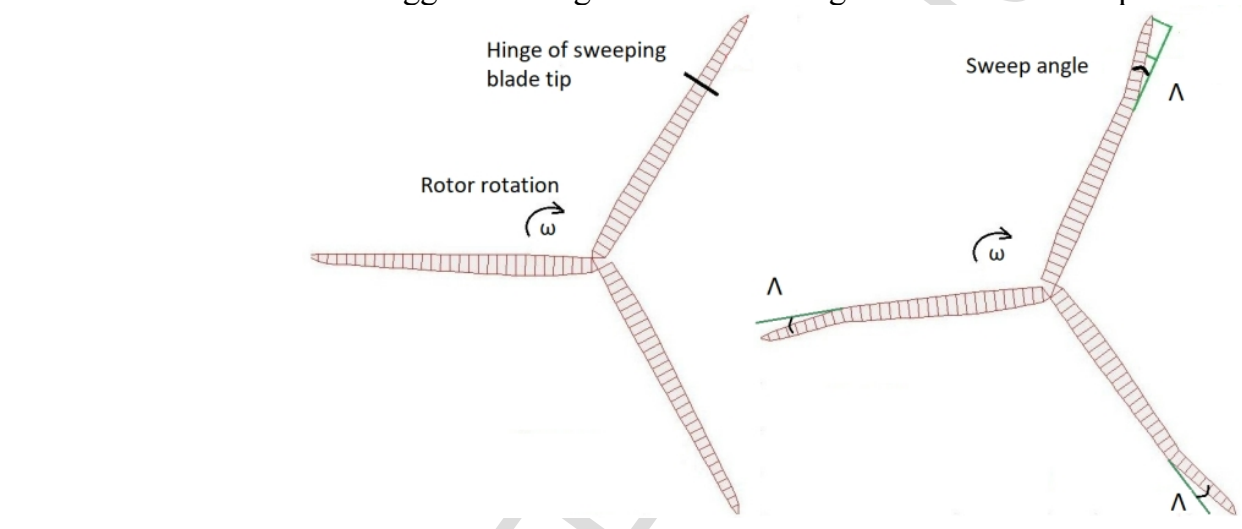

Fig. 1 Wind Turbine Rotor sweeping aft to compensate for a wind gust - Left: Sweep Angle $\Lambda=0$, Right: Sweep Angle $\Lambda$ in aft position

\section{METHODOLOGY}

\subsection{VORTEX METHOD}

The method used firstly in the present work in order to examine the effects of tip sweep is the Vortex method [5]. With this method the wing - or in this case the blade - is divided into small elements (also known as horseshoe elements) with all of its bound vorticity concentrated to the quarter chord and thus a refined model is introduced having span-wise distribution of bound circulation $\Gamma(\mathrm{y})$ [6], as shown in Figure 2. The bound circulation $\Gamma(y)$ is a measure of the fluid rotation (caused by wing's lift) at every element and in accordance with Kelvin Helmholtz theory these vortex lines (placed at the quarter chord) extend streamwise (in $\mathrm{x}$ direction) thus creating a vortex lattice which consists of shed and trailing vortices. 


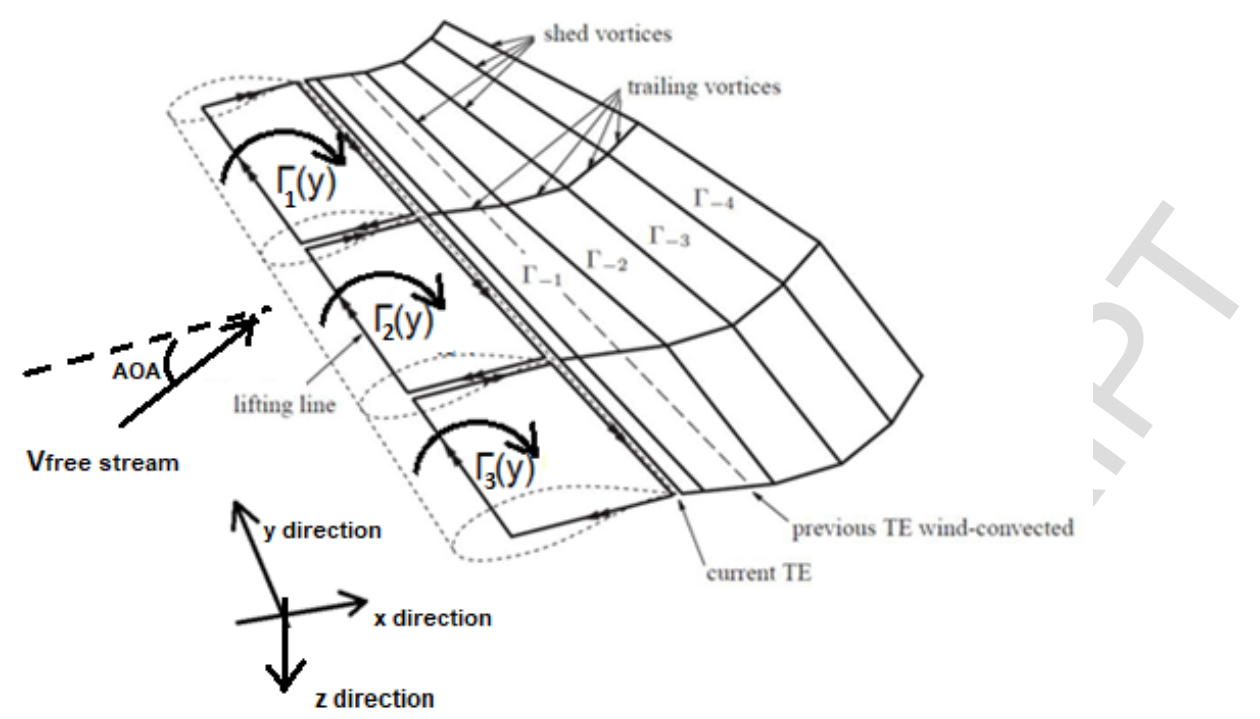

Fig. 2 : Lifting line vortex representation of a wing and its wake, source: [7].

The trailing vortices account for the span-wise bound circulation distribution $(\mathrm{d} \Gamma(\mathrm{y}) / \mathrm{dy})$ whereas the shed vortices account for the time rate of change of bound circulation ( $\mathrm{d} \Gamma / \mathrm{dt})$ (i.e. unsteady lift) and contain the history of the wing's lift force. This implies that in the steady state there are only trailing vortices and no shed vortices - except for the ones that were initially created and are located in the far wake. This vortex lattice in turn, creates a downwash on the blade which is expressed as induced velocity and can be calculated by using the Biot Savart law, which is formulated as:

$$
\overline{V_{i n d}}=\frac{\Gamma}{4 \pi} \frac{\left(r_{1}+r_{2}\right)\left(\overline{r_{1}} \times \overline{r_{2}}\right)}{r_{1} r_{2}\left(r_{1} r_{2}+\overline{r_{1}} \cdot \overline{r_{2}}\right)}
$$

where $r_{1}$ and $r_{2}$ are the distances of the vortex edges from the point where induced velocity is calculated, $\Gamma$ is the circulation of the straight segment and $V_{\text {ind }}$ is the induced velocity in a single point (by nearby vortex segments).

The induced velocities are calculated with the above formula at the so called, control points, which are located in the middle of every horseshoe element over the quarter chord. By superimposing induced velocities with free stream and blade section velocities (structure related or otherwise caused) results in a resultant velocity $\mathrm{V}\left(\mathrm{V}_{\text {resultant }}\right)$ for every section, which can be used directly in the three-dimensional form of Kutta-Joukowski equation (2) or for the determination of an effective angle of attack and finally extract lift, drag and moment coefficients by $2-\mathrm{D}$ steady state aerodynamic data. So, it is concluded that total wing forces and distributions of them are calculated straight from the vortex lattice.

$$
d \vec{F}=\rho \Gamma \vec{V} \times d \vec{l}
$$

\subsection{UNSTEADY LIFTING LINE THEORY - ULL}

One common use of lifting line theory is when unsteady flows or generally unsteady operating conditions are encountered. In this case the span-wise bound circulation distribution of the wing changes continuously in time and an iterative process is applied based on equation (2) which matches the bound circulation distribution with lift. Firstly the wing is divided into $i=1: N$ small elements as depicted in figure 2 and simulation time into $\mathrm{m}$ time steps where a guess is made about the wing's bound circulation distribution. Usually every time step starts with the distribution of the previous one. The trailing and shed vortices in turn are determined in accordance with equations (3) and (4). Since the vortex strength of all segments both from the wing and the wake is known (the wing vorticity derives from the initial guess and the wake vorticity has already been calculated from the previous time steps) the induced velocity, the resultant velocity and the effective angle of attack of every element are calculated. So, lift coefficient is 
acquired from tabular data and lifting force is exerted from equation (5) and a new bound circulation is determined from equation (2). Now, the bound circulation of the next step is given by equation (6) where an underelaxation factor is applied in order to prevent solution from diverging. This process is repeated until a user defined convergence criterion expressed with equation (7) has been obtained.

$$
\begin{gathered}
\left(\Gamma_{\text {Trail }}\right)_{i, m}=\left(\Gamma_{\text {Bound }}\right)_{i, m}-\left(\Gamma_{\text {Bound }}\right)_{i+1, m} \\
\left(\Gamma_{\text {Shed }}\right)_{i, m}=\left(\Gamma_{\text {Bound }}\right)_{i, m-1}-\left(\Gamma_{\text {Bound }}\right)_{i, m} \\
\text { Lift / span }=\frac{1}{2} \rho_{\infty} V_{\text {resul tan } t}{ }^{2} C_{L} c
\end{gathered}
$$

where:

- $C_{L}$ is the Lift coefficient

- $V_{\text {resultant }}$ is the sum of induced velocity, free stream velocity and blade section velocity (structure related or otherwise caused)

- $\quad \mathrm{c}$ is the chord of the wing element

- $\rho_{\infty}$ is the air density

$$
\Gamma_{\text {input }}=\Gamma_{\text {old }}+D\left(\Gamma_{\text {new }}-\Gamma_{\text {old }}\right)
$$

while $\max \left[\left(\Gamma_{\text {input }}-\Gamma_{\text {old }}\right) / \Gamma_{\text {old }}\right]>$ convergence_criterion

A Matlab code based on Unsteady Lifting Line Theory (ULL), is developed so as to study the effect of tip sweep on a fixed blade (non - rotating), both in steady and oscillatory conditions. The "hinge" of the sweeping part is placed in the quarter chord $(1 / 4 \mathrm{c})$ and occupies up to $30 \%$ of the total blade span. Quantities of interest are primarily lift and induced velocity distribution in $\mathrm{z}$ direction (generated from the vortex lattice of figure 2). The reasons that render this method suitable for this investigation are that wind turbine blades are of high aspect ratio which allows the accumulation of bound vorticity of the lifting surface on a single line and that wing geometric features like sweep or dihedral can be modelled quite accurately. Preliminary results referring to a fixed blade NACA 0012 with tip sweep, are compared to CFD simulations in ANSYS CFX [8] and good agreement is noticed [9].

\subsection{MODIFIED ULL}

As a next step, the ULL model is modified for the 5MW NREL reference wind turbine [10], where more quantities are investigated such as Power $\mathrm{P}$, Thrust $\mathrm{T}$ and Blade root Bending Moment $\mathrm{M}_{\mathrm{x}}$.

The role of tip sweep in the developed code is modeled according to the following considerations:

a. Lift coefficient of a swept wing is linked to the lift coefficient of the unswept wing with the equation (8).

$$
C_{L s w}=C_{L} * \cos ^{2}(\Lambda)
$$

b. The resultant velocity of the blade tip sections has an additional in-plane velocity due to tip movement .

c. The radial position of the blade tip sections is a function of tip sweep angle i.e. it is reduced for every sweep direction.

However, two approaches for the vortex lattice exist when using the lifting line method, the prescribed wake and the free wake evolution. With the prescribed method awareness of the wake development is needed a priori. Yet, it is orders of magnitude faster than the free wake approach when simulations are ran on computer based on corresponding algorithms. On the contrary, free wake approach lets the wake develop physically as a result of interactions between shed and trailing vortices of the vortex lattice. In particular, induced velocities are calculated from every vortex segment on every point of the lattice and after the addition of free stream velocity the convection of them is determined. The advantage with this method is that effects like wake distortion, vortex roll - up at the wingtip area and wake expansion are modeled which consequently leads to better predictions. The disadvantage on the other side is the high computational cost because of the large number of calculations needed for every lattice point that constantly grows in size as the wake unreels. In addition, stability problems on free wake algorithms can arise when wake points get close together due to singularities in the calculation of induced velocities. 
Therefore, a comparison between them is necessary, before proceeding to the next step utilize findings in lower fidelity such as BEM-based design codes.

In this work for those control points that are located close to vortex filaments, a cut-off radius is introduced to the filament and equation (1) is modified to equation (9). It is suggested by Van Garrel though, that for bound vortex calculations the cut off radius value should be about $0.01 \%$ of the vortex filament size [7] \& [11].

$$
\overline{V_{\text {ind }}}=\frac{\Gamma}{4 \pi} \frac{\left(r_{1}+r_{2}\right)\left(\overline{r_{1}} \times \overline{r_{2}}\right)}{r_{1} r_{2}\left(r_{1} r_{2}+\overline{r_{1}} \cdot \overline{r_{2}}\right)+\left(\delta_{r} l\right)^{2}}
$$

where: $\delta \mathrm{r}$ is percentage of vortex filament size 1 .
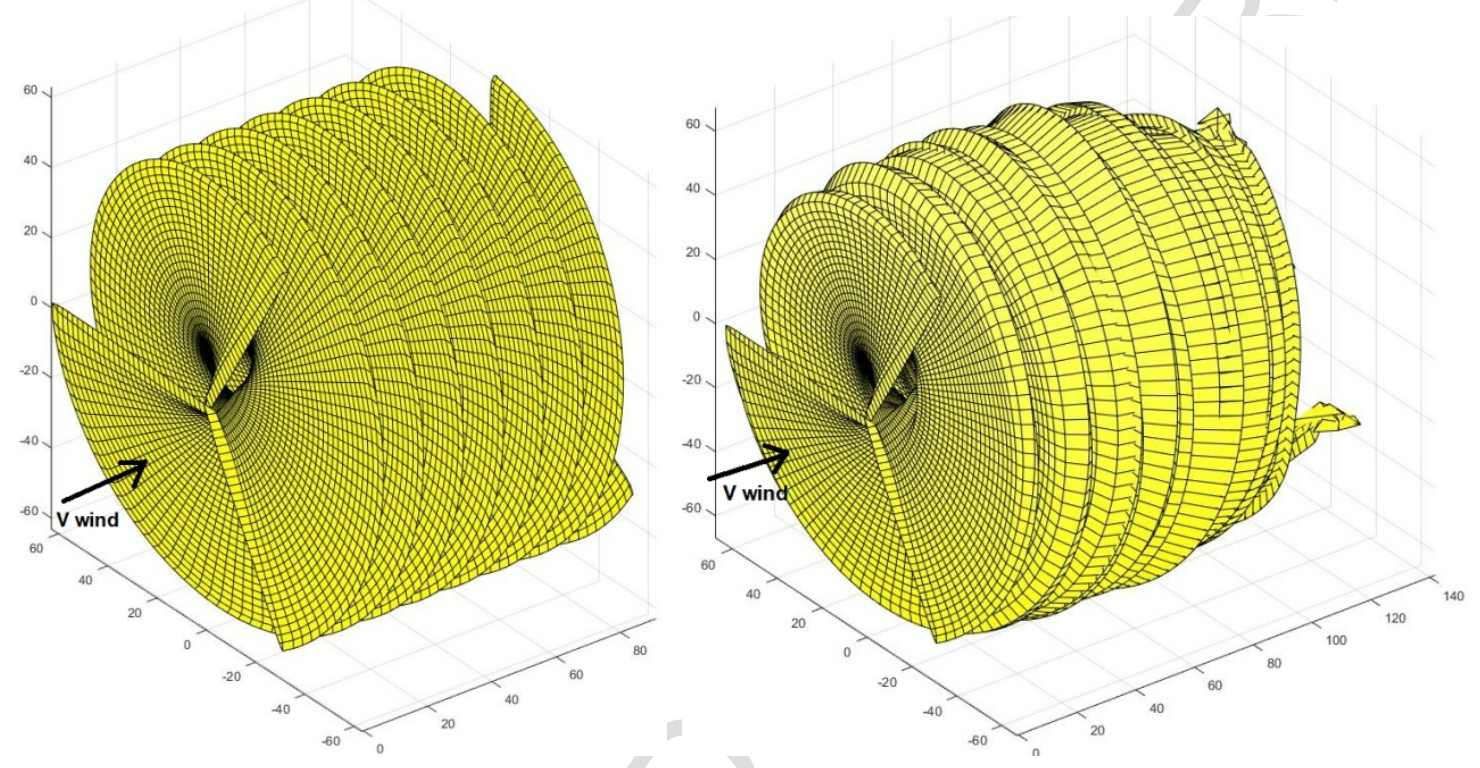

Fig. 3 : Prescribed wake development versus free wake development behind a 5MW NREL wind turbine rotor
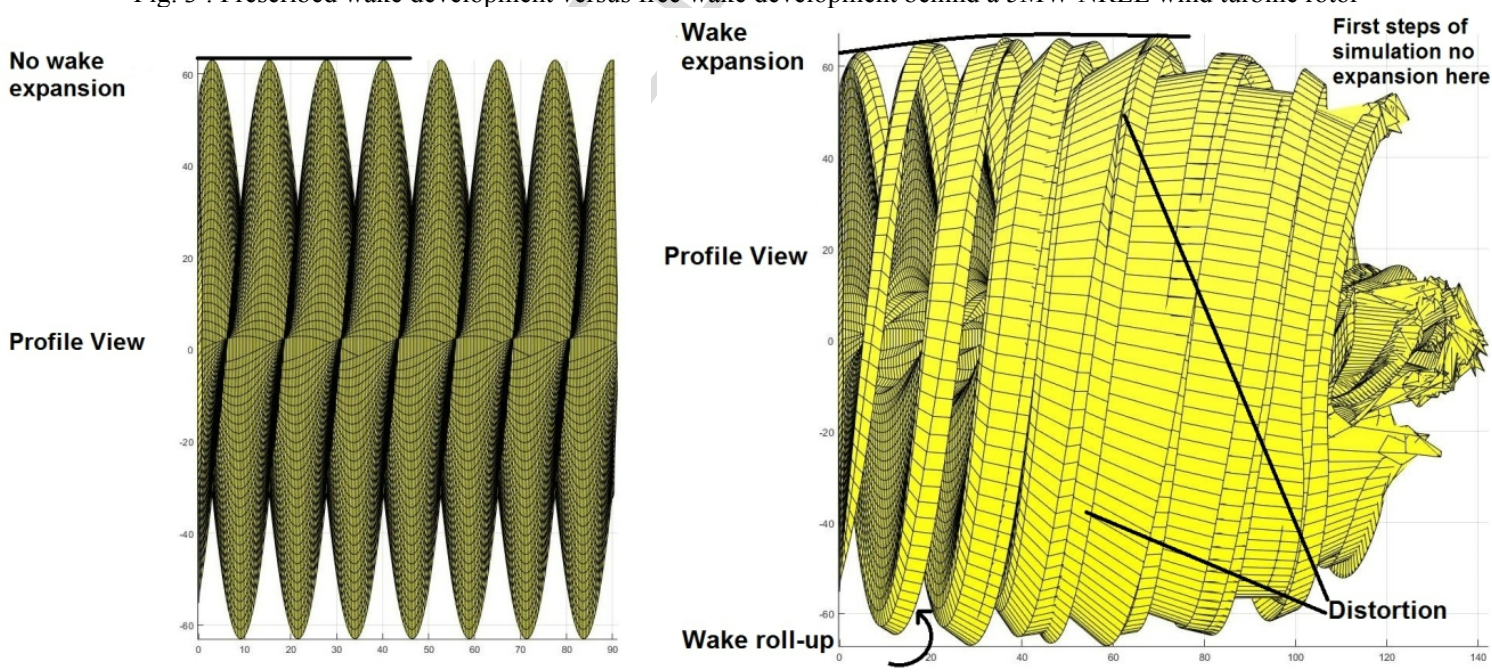

Fig. 4 : Prescribed wake development versus free wake development behind a 5MW NREL wind turbine rotor - profile view

Generally it is shown in [9], that the prescribed wake code (which has significantly lower demands in computational resources) agrees very well with the free wake code both in coarse parameters such as Power development and in considerable distributions such as blade induction. In this case, the wake nodes stem from the blades' trailing edge with velocity equal to $25 \%$ of the vectorial sum of free-stream and blade 
section velocity $\left(\omega^{*} \mathrm{r}\right)[7] \&[11]$. The rest of the nodes travel with $2 / 3$ of the free-stream velocity which is an assumption that defines optimal operating conditions for the wind turbine by using the optimal axial induction factor derived from momentum theory [11].

The results in [9], show that the main difference is expressed as an offset (of the order of 10\%) which is due to a faster moving wake calculated by the free wake code configuration. Similar differences in induction distributions are also seen in [11]. Besides the offset, it can be stated that the prescribed wake code configuration includes the wake roll-up effects, takes into account tip sweep both in steady - state and transient cases so its results can be used as reference for the purpose of developing a code with other theoretical basis such as BEM. Moreover, in line with the present trend for fast computations ULL codes are further modified to run on GPU utilizing NVIDIA CUDA platform [12] and its integrated support in Matlab and the process is accelerated up to 60 times.

Nowadays, the continuous progress in computer engineering has enabled the extended use of free wake codes and full rotor CFD so as to accurately calculate the blade aerodynamic loads. Nevertheless, BEMbased codes which are based on a different theory have evolved accordingly through specific improvements that take effectively into account trailing vorticity from the blades modeled by a tip loss factor, unsteady rotor wake dynamics modeled by a dynamic inflow model and unsteady airfoil aerodynamics modeled by Theodorsen theory [13]. A recent work [14] based on the Near Wake model originally proposed by Beddoes is a representative example of the current state of the art of high fidelity BEM models. Thus, the aforementioned evolution steps in combination with the indisputable low computational demands of these models (BEM - based models) render them still the first choice for research and industrial design applications.

The aim of present work is to develop a modified BEM code that accounts for rotor blades with variable tip sweep capability. The results of the already developed ULL model are used as a guide for this attempt. The next step is the addition of a suitable module that has the ability to control loads and power production for specific operating conditions.

\subsection{BLADE ELEMENT MOMENTUM THEORY - BEM}

BEM is a quite simple theory which combines the equations referring to the aerodynamic forces (Lift and Drag) produced by the blades with the equations referring to the momentum change of the flow which passes through the rotor. This results in the computation of two induction factors [equations (10) and (11)] on the rotor after an iterative process which are linked to the performance of the wind turbine.

where:

$$
a=\frac{1}{\frac{4 \sin ^{2} \phi}{\sigma C_{n}}+1}
$$

$$
a^{\prime}=\frac{1}{\frac{4 \sin \phi \cos \phi}{\sigma C_{t}}-1}
$$

- $\alpha$ is the axial induction factor showing how much loaded is the turbine

- $\quad \alpha^{\prime}$ is the tangential induction factor showing how much kinetic energy is lost through the addition of rotational speed to the wake

- $\varphi$ is the inflow angle (angle between $V_{\text {resultant }}$ and the rotor plane)

- $\mathrm{C}_{\mathrm{n}}$ is the force normal to the rotor plane (vectorial summation of Lift and Drag)

- $\mathrm{C}_{\mathrm{t}}$ is the force tangential to the rotor plane (vectorial summation of Lift and Drag)

- $\sigma$ is the solidity factor and expresses the fraction of annular area covered by rotor blades

This BEM model is a modification of the aerodynamic module of "DU_SWAMP_aero" [15] and incorporates the dynamic inflow model [16] in order to calculate the induced velocity of the wake. According to this, a filtering scheme is applied for the induced velocities, consisting of two first order differential equations (12) and (13). At first, the quasi steady value of the induced velocity is determined and then an intermediate value is calculated by applying a first order filter for the whole rotor. Eventually the induced velocity $\mathrm{W}$ is calculated by applying successively a second (first order) filter, which is a function of radial distance $r$ and ensures that the tip elements react faster than the root elements. The time constants $\tau_{1}$ and $\tau_{2}$ are calibrated with a simple vortex method [17] [equations (14) and (15)] 


$$
W_{\text {int }}+\tau_{1} \frac{d W_{\text {int }}}{d t}=W_{q s}+k \tau_{1} \frac{d W_{s}}{d t}
$$

$$
W+\tau_{2} \frac{d W}{d t}=W_{\text {int }}
$$

where:

- $\mathrm{W}$ is the calculated induced velocity

- $\quad \mathrm{W}_{\text {int }}$ is an intermediate value of the induced velocity

- $\cdot \mathrm{W}_{\mathrm{qs}}$ is the quasi steady value of the induced velocity

- $\quad \mathrm{k}$ is a constant and equals 0.6

- $\tau_{1}$ and $\tau_{2}$ are time constants

$$
\tau_{1}=\frac{1.1}{(1-1.3 \alpha)} \frac{R}{V_{0}}
$$

$$
\tau_{2}=\left(0.39-0.26\left(\frac{r}{R}\right)^{2}\right) \tau_{1}
$$

where $\mathrm{R}$ is the rotor diameter and $\mathrm{V}_{\mathrm{o}}$ is the inflow velocity far upstream of the rotor

In addition, the following adjustments were incorporated to the aforementioned BEM model:

a. The adoption of a refinement in the dynamic inflow model which considers an individual time constant for every radial distance $r$ and alters accordingly its axial induction value when dynamic phenomena set in. The factor $f$ is derived from equation (16) (ECN modeling) [18].

$$
f\left(\frac{r}{R}\right)=2 \pi / \int_{0}^{2 \pi} \frac{\left[1-r / R \cos \phi_{r}\right]}{\left[1+(r / R)^{2}-2 r / R \cos \phi_{r}\right]^{3 / 2}} d \phi_{r}
$$

where $r$ is the radial position, $R$ is rotor radius and $\phi_{r}$ is the rotor azimuth

b. All of the previously discussed modifications that were applied on the ULL codes for tip sweep consideration.

\section{BEM DEVELOPMENT STAGES}

\subsection{COMPARISON BETWEEN BEM AND ULL}

In order to verify that BEM accounts well for tip sweep considering raw parameters (Power, Thrust, Root Bending Moment) and distributions (axial induction factor) it is compared to ULL model with prescribed wake configuration at steady and unsteady conditions for the un-swept rotor. The following results are part of a wider research work which is found in [9] and therefore the most representative ones are presented here.

From the comparison in steady conditions, it is realized that there is a very good agreement between the two methods/models expressed mainly as an offset. The best agreement is observed for the rotor thrust $\mathrm{T}$ (under 1\%) which stands for the out-of-plane forces. Nevertheless, the in-plane forces that are responsible for power generation are also modeled well by BEM creating a relative difference under $5 \%$. The two methods show a slightly different transient response to steady state, due to the particular modelling of wake dynamics. As far as the axial induction distribution is concerned the average relative difference is $7 \%$ at the mid blade area but at the root and tip area the disagreement is noteworthy which is due to the trail vorticies that are calculated better by ULL. This difference in the tip area where torque is the greatest is the main reason for the power difference between BEM and ULL.

Figures 5 and 6 show the results which are obtained from simulations at the same flow conditions and blade configurations for the two different models - ULL (prescribed wake) and BEM. Figure 5 shows the power variation as a function of time, and Figure 6 the blade out-of-plane root bending moment variation of a $5 \mathrm{MW}$ NREL rotor operating at rated conditions [Vwind $=11.4 \mathrm{~m} / \mathrm{s}$ (as shown in figure 3 ) and $\omega=1.26 \mathrm{rad} / \mathrm{s}$ ] which is equipped with $20 \%$ tip swept blades, (i.e. with a length measuring $20 \%$ of the total blade span). The blade tips are subjected to a harmonic sweep angle oscillation through an actuator and the effect of this scheme is shown. The amplitude of sweep angle variation is \pm 12 degrees and the frequency $\mathrm{f}$ is $0.125 \mathrm{~Hz}$ and $0.25 \mathrm{~Hz}$. The oscillation starts from the un-swept position with aft direction. In addition, the blades are 
considered rigid and only the aerodynamic forces are examined. This comparison shows how well (compared to ULL theory) BEM calculates loads in unsteady operating conditions.

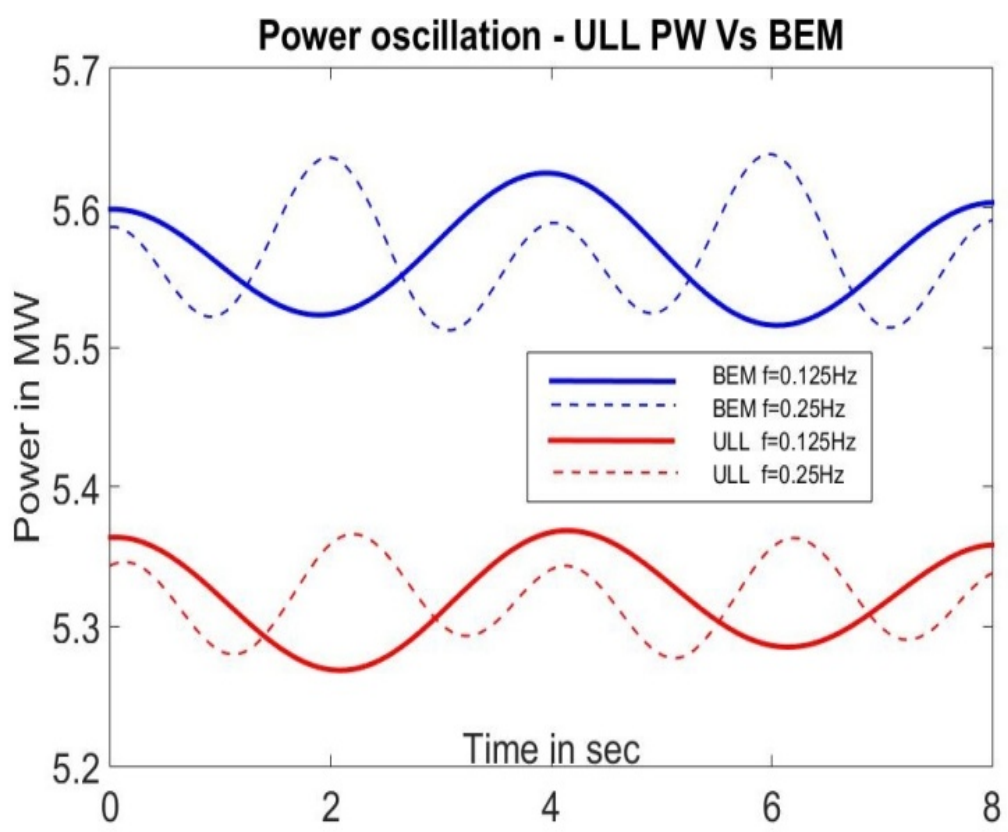

Fig. 5 Comparative diagram of power oscillation for the $20 \%$ tip swept NREL 5MW rotor predicted by ULL and BEM. Amplitude $=12 \mathrm{deg}$ - frequency $=0.125$ and $0.25 \mathrm{~Hz}-\mathrm{t}=0$ tip starts with aft sweep.

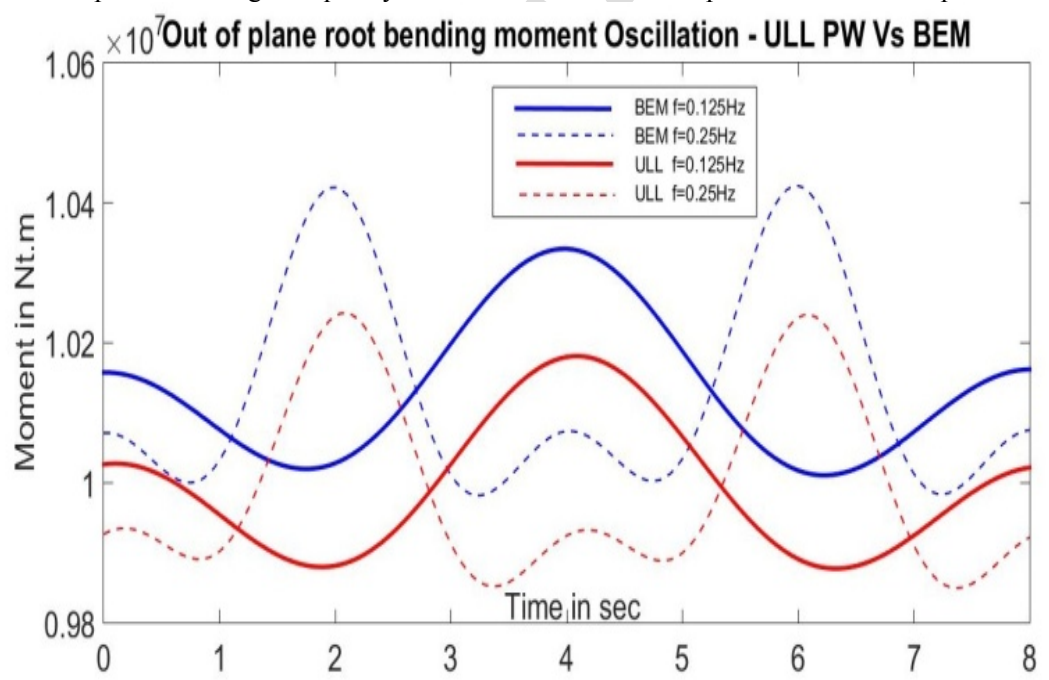

Fig. 6 Comparative diagram of Blade 1 root bending moment oscillation for the $20 \%$ tip swept NREL $5 \mathrm{MW}$ rotor predicted by $\mathrm{ULL}$ and BEM. Amplitude $=12 \mathrm{deg}$ - frequency $=0.125$ and $0.25 \mathrm{~Hz}-\mathrm{t}=0$ tip starts with aft sweep.

It is noticed that BEM and ULL produce an almost identical dynamic behavior for unsteady conditions and once again a constant offset between the values is observed. Both methods uncover the increasing effect of additional tip velocity with increasing oscillating frequency which is clearly seen when comparing the two un-swept positions $\mathrm{t}=2 \mathrm{sec}$ and $\mathrm{t}=4 \mathrm{sec}$ for the $\mathrm{f}=0.25 \mathrm{~Hz}$ case. In this case the additional velocity does not exceed $3.7 \mathrm{~m} / \mathrm{s}$ at the blade tip.

\subsection{THE BEM "PROBLEM"}

Despite the fact that a relatively good agreement is observed between BEM and ULL model in terms of coarse parameters such as Power and Total rotor thrust, a different picture is obtained in the calculation of 
the axial induction factor distribution. In particular, the BEM model does not predict the characteristic kink in the tip area (where the ULL model does), when the blade tip is swept backwards, as it is seen in figure 7. In particular, Figure 7 depicts the steady state results from two simulations performed with the two different models (ULL and BEM). In this case the axial induction distribution is shown for a 5MW NREL rotor operating at rated conditions (Vwind $=11.4 \mathrm{~m} / \mathrm{s}$ and $\omega=1.26 \mathrm{rad} / \mathrm{s}$ ) which is equipped with $10 \%$ tip swept blades that are given a 20 degree aft sweep angle.

Therefore, a correction should be adopted in the module that calculates the Prandtl's tip loss factor in order to account for tip sweep. The explanation about this discrepancy is the fact that BEM is based on the assumption for radial independence and as such, the trailed vorticity caused by the sweep angle variation is not considered.

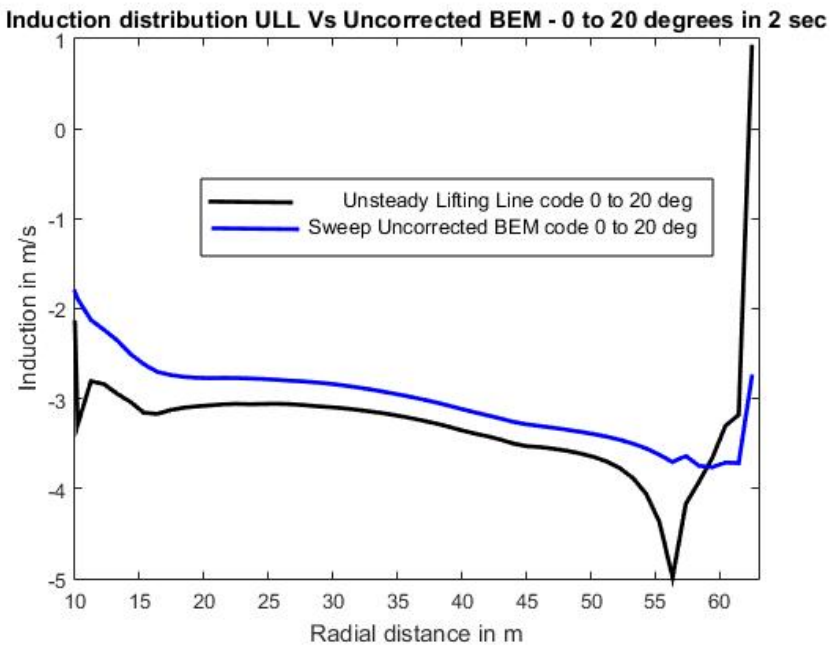

Fig. 7 Comparative diagram of induction distribution for the $10 \%$ tip aft swept at 20 degrees NREL 5MW rotor predicted by ULL and BEM.

\subsection{BEM CORRECTION}

One choice for correction could be the establishment of a new theoretical model which is based on the new position of the emanating trailing vorticities as the blade tip sweeps aft and develop new factors or new time constants that affect the induction distribution of the blade. A representative example is the further development of the near wake model originally proposed by Beddoes and the coupling of it with a far wake model [14] to provide a better tip loss correction. However, in this work the aim is to develop an engineering model which is fast, effective and collaborates well with the current BEM code configuration. So, it is important to introduce a parameter that is already calculated in the BEM model and changes according to sweep angle. A suitable parameter for the sweep correction is the radial bound circulation difference distribution $(\mathrm{d} \Gamma(\mathrm{r}+1)-\mathrm{d} \Gamma(\mathrm{r}))$ and this is depicted below in figure 8 . Figure 8 depicts the steady state results from two simulations performed only with the ULL model. In this case the radial bound circulation difference distribution $(\mathrm{d} \Gamma(\mathrm{r}+1)-\mathrm{d} \Gamma(\mathrm{r}))$ is shown for a 5MW NREL rotor operating at rated conditions (Vwind $=11.4 \mathrm{~m} / \mathrm{s}$ and $\omega=1.26 \mathrm{rad} / \mathrm{s}$ ) which is equipped with $10 \%$ tip swept blades that are given a 20 degree aft sweep angle. 


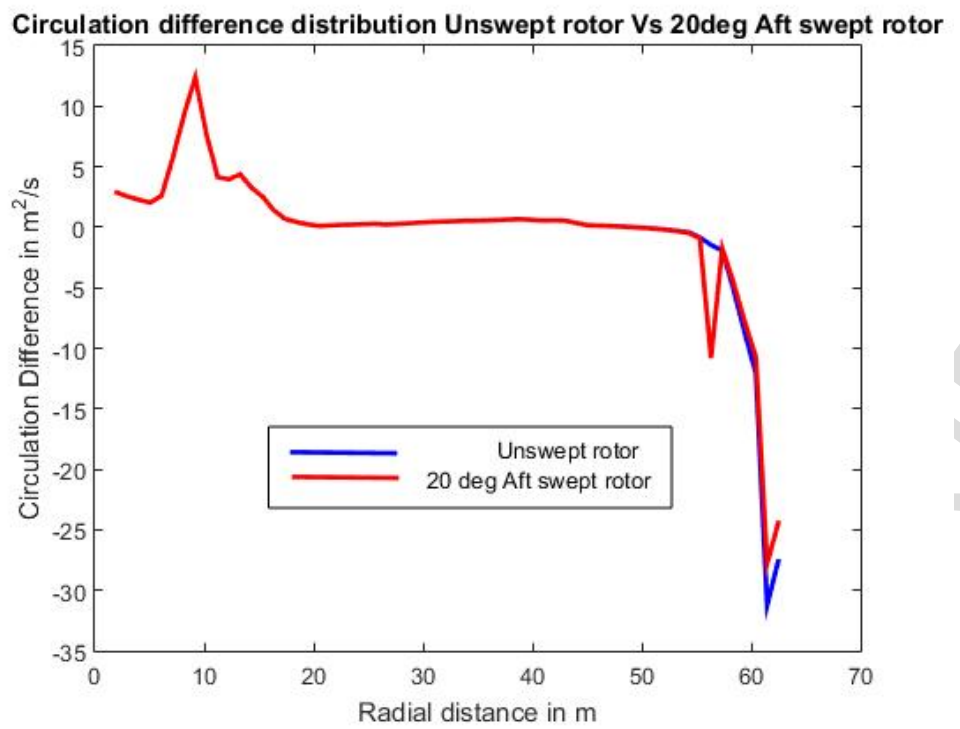

Fig. 8 Radial bound circulation difference for Un-swept and 20deg Aft swept rotor - 10\% sweep percentage.

The above figure indicates that a "kink" is formed in the distributions of circulation difference as the blade tip sweeps aft and it is located at the hinge area. The same "kink" is discovered in figure 7 and thus, the induction distribution of the BEM code can be corrected utilizing this fact. However it seems that improvement can be pursued mainly on this small part of the blade because changes in circulation difference are not extended to the rest of the tip. So, an additional consideration for the rest of the blade tip should be made. A suitable parameter that changes noticeably as the tip sweeps aft is the distance travelled by the swept part of the blade in the plane of rotation. This distance is proportional to the distance of the blade element from the hinge and expresses the potential of the tip vortex as it changes stemming position.

The philosophy of the adopted correction in the induction distribution is based on the Biot Savart formula equation (1) that is already used to calculate the induction in the ULL model.

The proposed correction consists of two parts - the first focuses on the hinge area and the second on the rest of the blade tip. Equation 17 presents the general form of the proposed correction expressed by the factor $\mathrm{G}$ :

$$
G=-a g_{1} \operatorname{Circ}_{\text {diff }} /\left(V_{\text {inf low }} 4 \pi d r^{2}\right)-b g_{2} X_{C p G}{ }^{2} \operatorname{Circ}_{\text {diff }} /\left(\left(V_{\text {inf low }} 4 \pi d r^{4}\right) \quad \text { (non }-\right. \text { dimensional) }
$$

where:

- $\quad \alpha, b$ : factors that accrue from tests and adjust the correction

- $\mathrm{g}_{1}, \mathrm{~g}_{2}$ : factors that maximize the correction at the hinge area $\left(\mathrm{g}_{1}\right)$ and also amplify the correction at the blade tip area $\left(\mathrm{g}_{2}\right)$. The values of this factors accrue from the normal distribution curves of the blades' elements radial distances. $g_{1}$ factor results from this normal distribution shifted to the hinge area and $\mathrm{g}_{2}$ shifted to the tip area accordingly.

- Circ diff : the bound circulation difference between adjacent blade elements in other words the trailed vorticity. (the value of the outermost circulation difference is the subtraction of the tip element bound circulation with zero)

- $X_{C p G}$ : is the vertical distance travelled in - plane by the blade elements according to the sweep angle of the blade tip (in relation to the unswept blade)

- $\mathrm{dr}$ : the blade element length

The $\mathrm{G}$ factor of equation (16) is calculated for every blade element of all rotor blades and is applied directly to the already calculated and corrected for the tip loss phenomena axial induction factor in the form of:

$$
\mathrm{a}_{\text {if }}=\mathrm{a}_{\text {if }}(1+G)
$$

However, in order to establish a correction that accounts only for the blade tip sweeping and thus would not interfere in the axial induction factor calculation when the blades remain un-swept factor $\mathrm{k}$ is subtracted from $\mathrm{G}(\mathrm{Eq} 19)$. i.e. 


$$
\mathrm{a}_{\mathrm{if}}=\mathrm{a}_{\mathrm{if}}(1+G-k)
$$

The $\mathrm{k}$ factor is calculated from equation 16 with the only difference that "Circ_diff" is the bound circulation difference between adjacent blade elements as if the blades are unswept. So on one hand, there is no correction when the blades are un-swept because $\mathrm{G}=\mathrm{k}$ and on the other hand correction is applied to the $\mathrm{a}_{\text {if }}$ only when the blades sweep. In this case the correction results from the difference in the trailed vorticity of the current blade configuration in relation to the trailed vorticity for the un-swept configuration $\mathrm{G} \neq \mathrm{k}$.

The application of the correction yields improved results for the $\mathrm{a}_{\mathrm{if}}$ distribution, with respect to the un modified BEM model, as it seen in figure 9. In particular, Figure 9 depicts the steady state results from two simulations two simulations performed only with the BEM model - modified and unmodified. In this case the axial induction distribution is shown for a 5MW NREL rotor operating at rated conditions (Vwind $=11.4 \mathrm{~m} / \mathrm{s}$ and $\omega=1.26 \mathrm{rad} / \mathrm{s}$ ) which is equipped with $10 \%$ tip swept blades that are given a 20 degree aft sweep angle. The characteristic kink is formed and the tip area is affected accordingly.

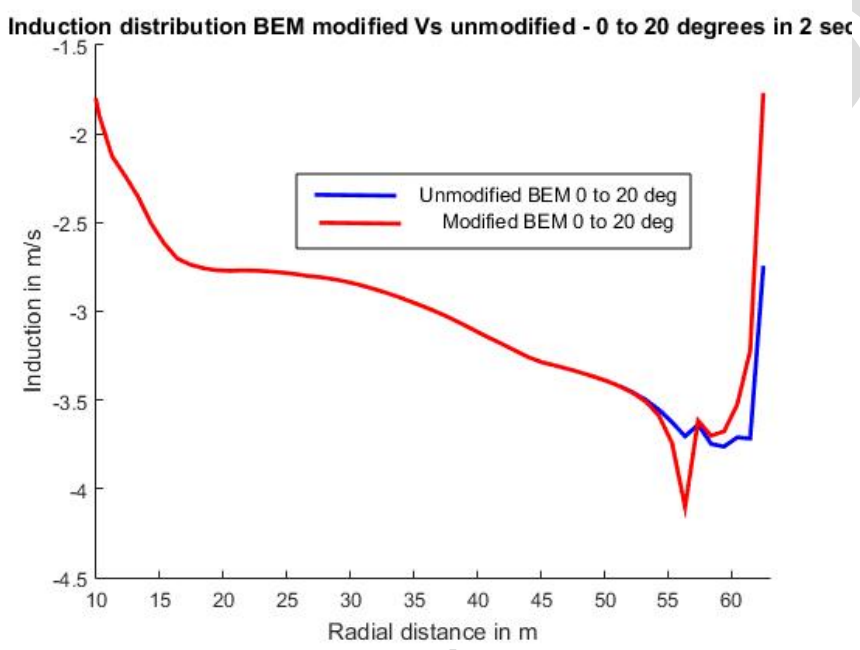

Fig. 9 Induction distribution curves for sweep modified and unmodified BEM code 20 deg aft sweep 10\% tip sweep

The impact of this correction to the wind turbine coarse parameters, however is small. The out of plane forces practically remain unaltered whereas Power estimations change in the order of $0.25 \%$ both for fixed and active blade tip configurations. The influence in power is small but can become more significant in the calculation of the AEP for rotors with variable tip sweep capability.

\section{PARAMETRIC STUDY OF TIP SWEEP INFLUENCE ON WIND TURBINE QUANTITIES WITH ULL MODEL}

In order to investigate the potential of variable blade tip sweep concept as an active control method, a parametric study is performed for different blade tip sweep percentages that range from $10 \%$ to $30 \%$ of the total span. The simulations are performed on the prescribed wake version of the ULL code and focus on the harmonic tip motion of equation (20):

$$
\Lambda=\Lambda_{0} \sin \omega t
$$

where:

- $\Lambda$ is the sweep angle of the swept tip.

- $\Lambda_{0}$ is the amplitude of the harmonic motion.

- $\omega$ is the angular velocity of the swept part and equals to $2 \pi \mathrm{f}$.

The amplitude $\Lambda_{0}$ is set to 10degrees and the frequency f of sweeping motion is set to $0.125,0.25$ and 0.5 $\mathrm{Hz}$. The effect of sweeping motion on three basic wind turbine quantities, Power, Thrust and Blade No1 Root Bending moment, is addressed through the non-dimensional variable $\mathrm{N}_{\text {ratio }}$ against the nondimensional variable of $\mathrm{f}_{\text {ratio }}$ defined by equations (21) and (22) accordingly. 


$$
N_{\text {ratio }}=\frac{A m p(x)_{s}}{(X)_{0}}
$$

where:

- $\operatorname{Amp}(\mathrm{x})_{\mathrm{s}} \operatorname{Amp} \mathrm{x}$ is the amplitude of the examined $\mathrm{x}$ quantity (power, total thrust or root bending moment of blade No1) that results from the harmonic tip motion.

- $\mathrm{X}$ is the value of the same quantity in stable conditions $(11.4 \mathrm{~m} / \mathrm{s}$ wind speed in this case) and un swept blade configuration $(\Lambda=0)$.

$$
f_{\text {ratio }}=\frac{f_{\text {sw }}}{f_{\text {rot }}}
$$

where:

- $\mathrm{f}_{\mathrm{sw}}$ is the frequency of the sweeping motion of the blade tip.

- $\mathrm{f}_{\mathrm{rot}}$ is the (rotational) frequency of the rotor.

Figure 10 presents the effect of harmonic sweeping motion of the blade tip on three basic wind turbine parameters and figures 11 and 12 show the same effect individually, on Power and blade No1 root bending moment. The results in each of the following 3 figures are obtained from 9 simulations with the ULL model for the 5MW NREL turbine operating at rated conditions (Vwind $=11.4 \mathrm{~m} / \mathrm{s}$ and $\omega=1.26 \mathrm{rad} / \mathrm{s}$ ). The surface plot refers to 3 individual blade configurations (tip sweep percentage) and 3 different sweep angle oscillating frequencies. The amplitude of sweep angle variation is kept constant to 10degrees.

\section{Nratio VS fratio for Power, Thrust, Blade Root Bending Moment}

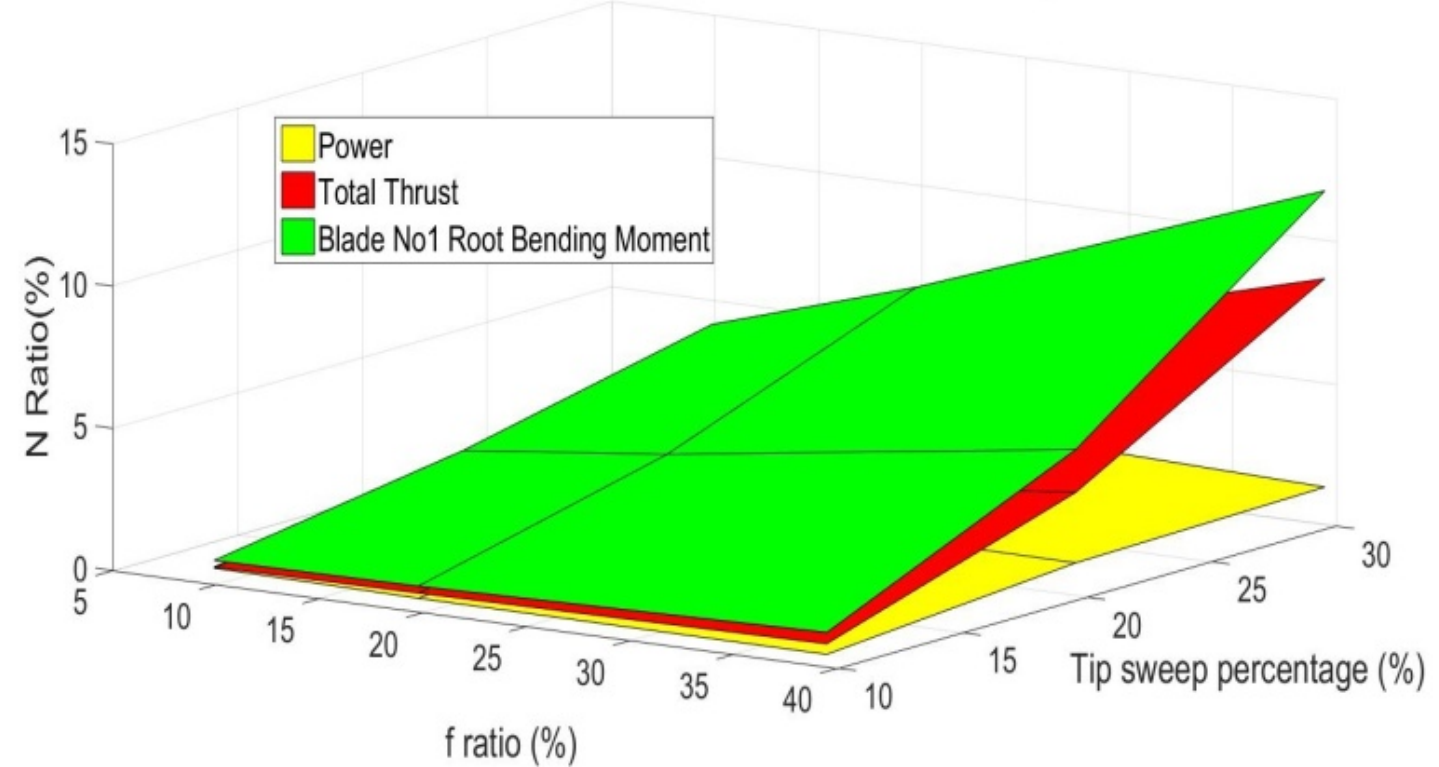

Fig. 10 Nratio vs fratio for Power Total thrust and Blade No1 root bending moment concerning a $10 \%, 20 \%, 30 \%$ tip swept rotor at rated operation $(\mathrm{V}=11.4 \mathrm{~m} / \mathrm{s})$ and sweep angle variation according to $\Lambda=10 \sin (\omega \mathrm{t})$ for $\omega=\mathrm{pi} / 4$, pi $/ 2$, pi 


\section{$\mathrm{N}$ ratio VS f ratio for Power}

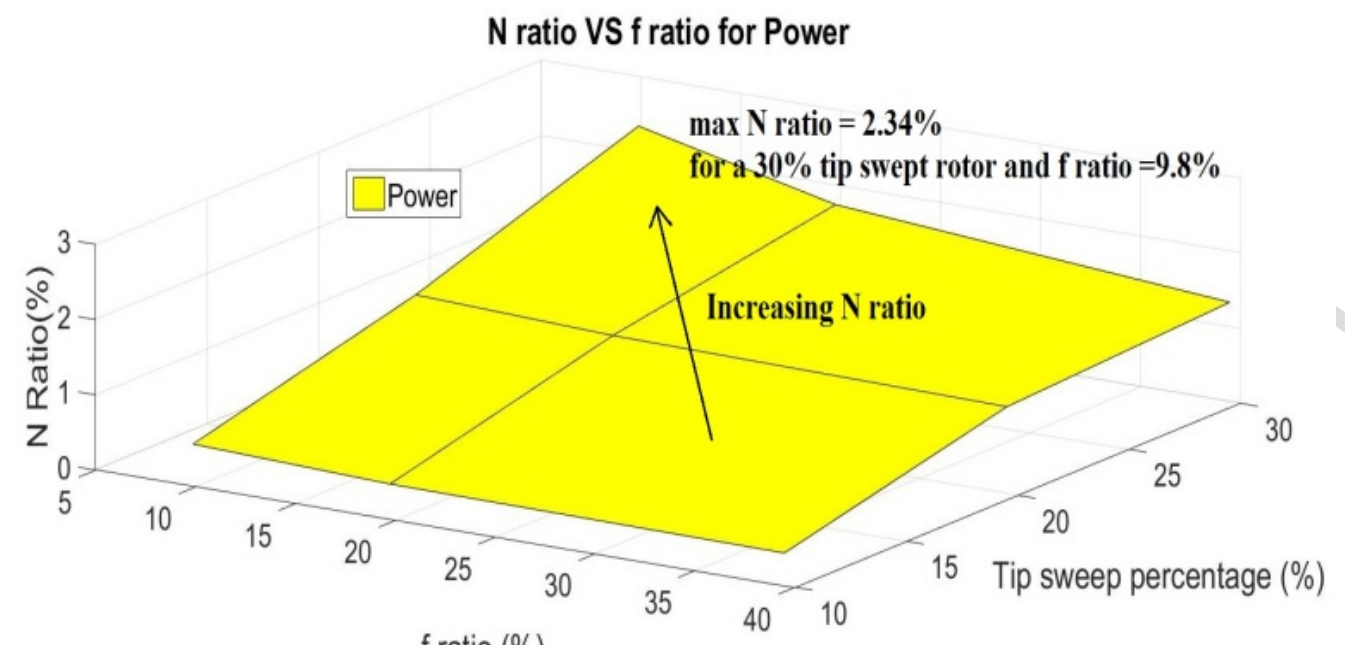

f ratio $(\%)$

Fig. 11 Nratio vs fratio for Power concerning a 10\%, 20\%, 30\% tip swept rotor at rated operation ( $\mathrm{V}=11.4 \mathrm{~m} / \mathrm{s})$ and sweep angle variation according to $\Lambda=10 \sin (\omega \mathrm{t})$ for $\omega=\mathrm{pi} / 4, \mathrm{pi} / 2$, pi

\section{$\mathrm{N}$ ratio VS $\mathrm{f}$ ratio for Blade No1 Root Bending Moment}

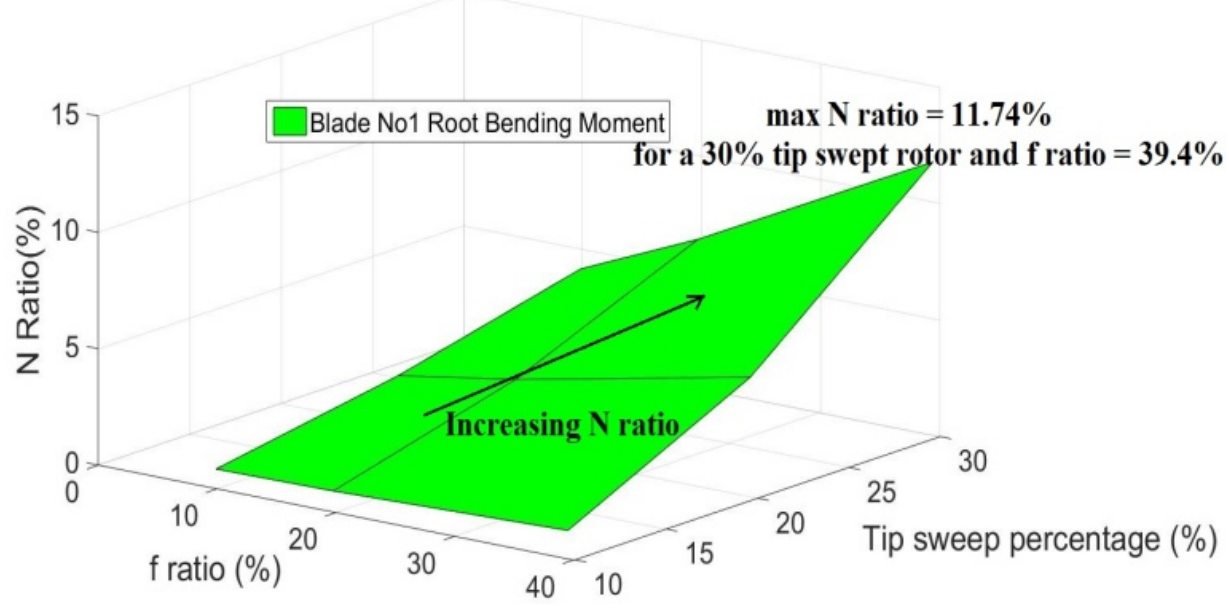

Fig. 12 Nratio vs fratio for Blade No1 root bending moment concerning a 10\%, 20\%, 30\% tip swept rotor at rated operation $(\mathrm{V}=11.4 \mathrm{~m} / \mathrm{s})$ and sweep angle variation according to $\Lambda=10 \sin (\omega \mathrm{t})$ for $\omega=\mathrm{pi} / 4, \mathrm{pi} / 2$, pi

As expected, every parameter is affected from the sweeping motion, with Blade No1 root bending moment being the most affected one. In addition, power seems to have a different behavior compared to Blade root bending moment that is, $\mathrm{N}_{\text {ratio }}$ increases in an opposite direction. In particular as sweeping motion increases in frequency, the amplitude of power decreases. However, all quantities increase with higher tip sweep percentage of the rotor blades. The maximum $\mathrm{N}_{\text {ratio }}$ value for every parameter is accordingly $2.34 \%$ and $11.74 \%$ for a $30 \%$ tip swept rotor and $\mathrm{f}_{\text {ratio }} 39.4 \%$ - namely for a sweeping motion frequency about half the frequency of the rotor.

Thus, it is concluded that tip swept rotors have a higher impact on out of plane loads (namely blade root bending moment) rather than in plane loads (namely power). So, if variable blade tip sweep is to be developed as a control feature it is presumed that it would be more suitable for load reduction rather than power improvement.

\section{ACTIVE BLADE TIP SWEEP CONTROL METHOD}

The objective of this study is to increase power production through blade extension, regulate fatigue loads during a high turbulent wind input and reduce extreme loads during an extreme wind gust by 
sweeping actively the rotor blade tips. This is attempted with a BEM based model that takes efficiently into account the effect of blade tip sweep and incorporates a suitable controller.

The first goal of Power production (AEP) increase, cannot be achieved by just incorporating a sweep angle active controller. This was deduced from the parametric study performed within [9]. Backward sweeping reduces loads and in addition, power production. Power production could theoretically be achieved through sweep angle variations that bring the $a_{\text {if }}$ distribution closer to the value of 0.3 where $C_{P}$ maximizes [19]. However, it is found from corresponding simulations, that the axial induction factor does not have a unidirectional change along the span of the swept part as sweep angle changes. So, the option of increasing the rotor swept area A by increasing the blade span is considered in this research work. The increased loads that follow the increase in blade span in counteracted by sweeping the tip aft. This is similar to the STAR (sweep - twist adaptive rotor) development program which is the most representative implementation of the geometric feature of sweep in wind turbines [4].

On the other hand, load reduction (due to turbulence or wind gusts) could be achieved with a suitable active controller. The developed control module, is based on a closed loop feedback system that monitors the average root bending moment of the 3 blades $M_{x} 1, M_{x} 2, M_{x} 3$ which then passes to a high pass filter (HPF) where only the dynamic effects are included. Then, this value is multiplied with a "to be determined - optimized" gain K which commands an actuator to sweep the blades at specific angle values. Sweep commands are filtered by a low pass filter with a time constant of $0.5 \mathrm{~s}$ to simulate a realistic response of a mechanical tip sweep system. Of course a careful study on the structural and dynamic characteristics of an actuator that should pivot the blade tips to the desired sweep angle will specify this parameter. In addition, a separate module maintains a stable operation during the start up phase in which the controller behaves aggressively because of the large gradients that appear. Lastly in the optimization phase, the controller's HPF time constant and controller gain $\mathrm{K}$ for which the interquartile value of blade load is minimized during a wind gust (within the sweep angle constraints), are found. The latter is accomplished with the use of the response optimization toolbox in Matlab and Figure 13 illustrates the aforementioned control scheme. Therefore it is clarified that the optimization process already described, is achieved offline for certain wind turbine class. If another class is used optimization should be redone as the gust according to which the optimization was performed will change. In paragraph 6.3 a more detailed reference regarding the extreme gusts is outlined.

At last, it has to be mentioned that in the case of a rigid rotor where only aerodynamics are included, the active controller is always stable because aft sweeping only reduces loads. Its stability has to be checked again when aeroelastics are included and sweep angle changes from the controller could induce blade deformations that will finally lead to raise of aerodynamic loads.

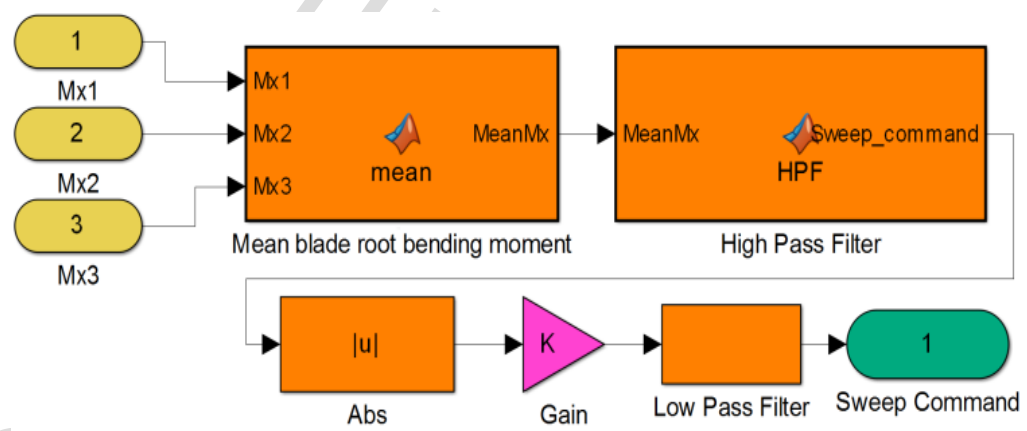

Fig. 13 The control method for load reduction in Matlab Simulink- $\mathrm{M}_{\mathrm{x}} 1, \mathrm{M}_{\mathrm{x}} 2, \mathrm{M}_{\mathrm{x}} 3$ are the root bending moments of the 3 blades

\section{RESULTS AND DISCUSSION}

The results of the investigation are presented with the following order: Power production increase, load alleviation during high turbulence winds and during wind gusts.

\subsection{POWER PRODUCTION}

Although power production increase is not achieved with an active control method, the idea of increasing rotor diameter along with the introduction of a constant backward sweep at the tip area leads to 
favorable results. After a parametric study [9], in order to determine the best configuration of the NREL blades an increase of $4.28 \%$ in AEP value is calculated along with a $2.77 \%$ increase for the maximum blade root bending moment loads. The new wind turbine design is configured with $5 \%$ extended blades that are swept backward 30 degrees. The tip sweep percentage is $10 \%$ of the overall blade span and the chord twist distributions of the new blade design are shown in figures 14 and 15 as a result from the parametric study. It is mentioned that only the blade tip geometry is modified in the presented designs where the rest of the blade remain unaltered.

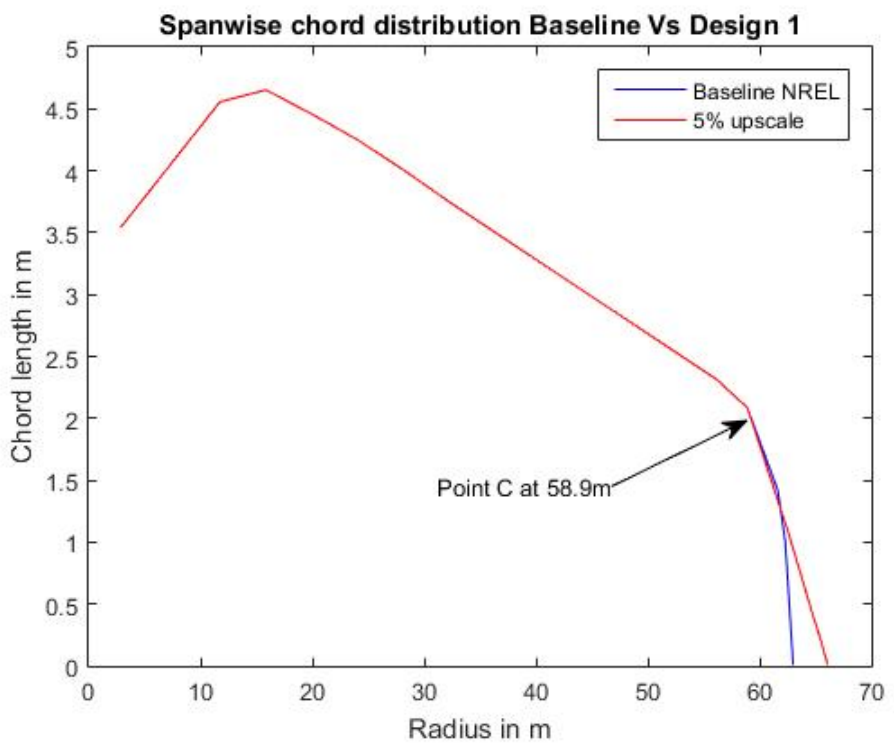

Fig. 14 NREL Vs New Blade design chord length distribution

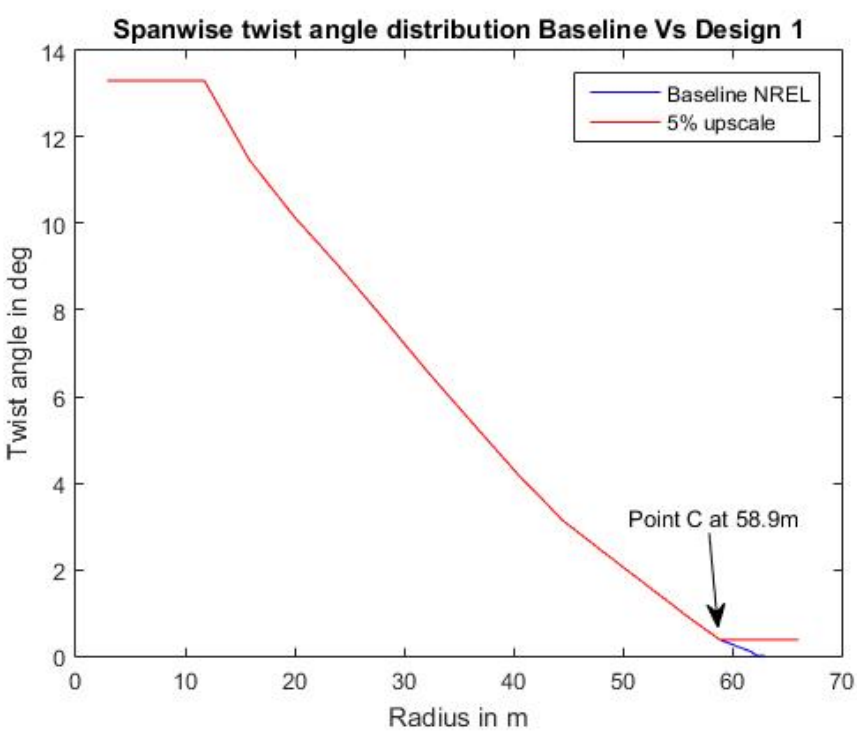

Fig. 15 NREL Vs New Blade design twist angle distribution

The proposed design as formulated here with the aforementioned parameters, is considered a feasible concept in terms of providing increased power production with a small load penalty in nominal operation that does not impose any significant structural reinforcements for the wind turbine.

\subsection{LOAD ALLEVIATION}

In the present section we attempt to portray the merits of this control concept to wind turbine rotors of this class (NREL 5MW 63m radius) with regard to load alleviation. Fatigue load reduction is the main 
motivation for almost every new control concept as they are intended for use in large wind turbine rotors where further unsteady load reduction is required. The benefits from load alleviation are translated either to extended service life of the wind turbine or lower production cost through lighter components. In this work, fatigue load reduction is investigated through a variable tip swept wind turbine rotor with the aforementioned recommended design ( $5 \%$ span extension $10 \%$ tip sweep percentage).

The following results refer to the rated power production case of a 3-D turbulent wind input field with $10 \%$ turbulent intensity "I" according to Kaimal spectrum and average wind speed of $11.4 \mathrm{~m} / \mathrm{s}$ that is seen by the modified wind turbine. The wind field is generated in TurbSim [20] software and figure 16 represents the three wind velocity components for 650 secs.
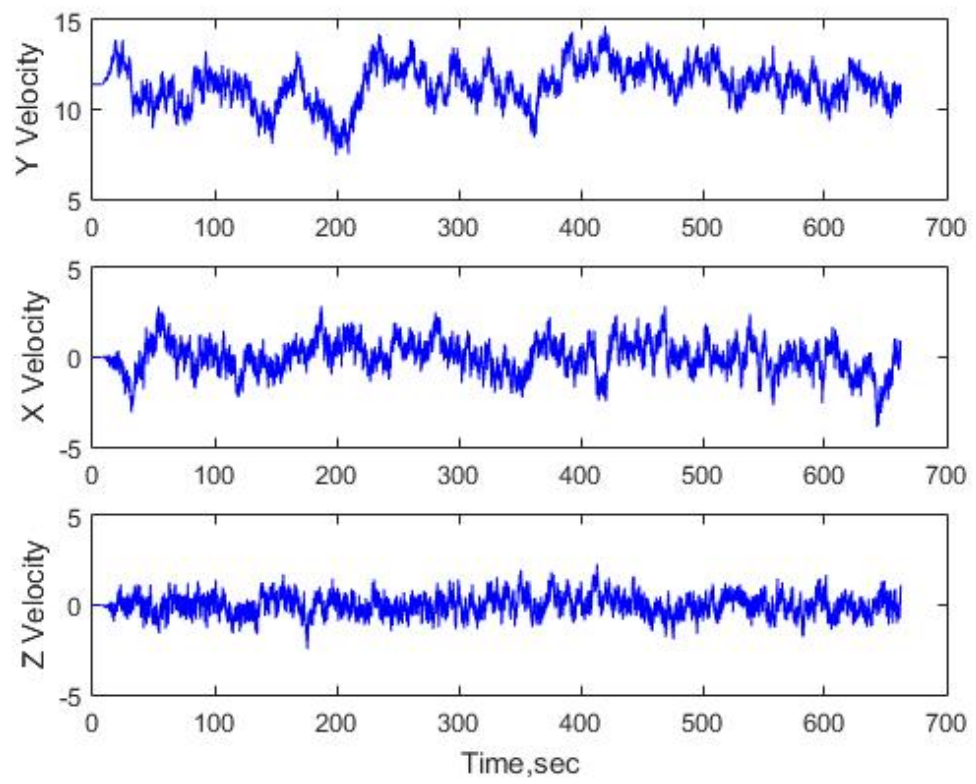

Fig. 16 The three wind velocity components at hub height for the examined test case

In figure 17 it is seen in practice, how the controller responds to the unsteady wind environment of figure 16 . The results derive from simulation runs for the modified 5MW NREL wind turbine $(5 \%$ span extension $10 \%$ tip sweep percentage) that incorporates active sweep control using the BEM model. The controller operates constantly as a response to the unsteady wind input with a maximum tip sweep angle command of 18 degrees. In addition, the maximum additional tip speed (outmost blade element) due to the controller commands, is $2 \mathrm{~m} / \mathrm{s}$ which means that there are not any abrupt changes that are translated to high inertia loading of the control mechanism.

In figure 18 (same simulation runs using BEM) the root bending moment of blade 1 is shown with and without the controller and it is seen that it operates in a way that lowers the peak loads during the turbulent wind input but it is not as effective during the load "valleys". This is explained by the controller settings (responds when loads increase) and generally by the operating principle of the tip sweeping concept; aft tip sweeping only decreases the loads for any sweep angle and thus when loads are decreased for other reason - in this case wind speed drops - the controller is not capable of trimming the corresponding valleys through sweep angle variations. 

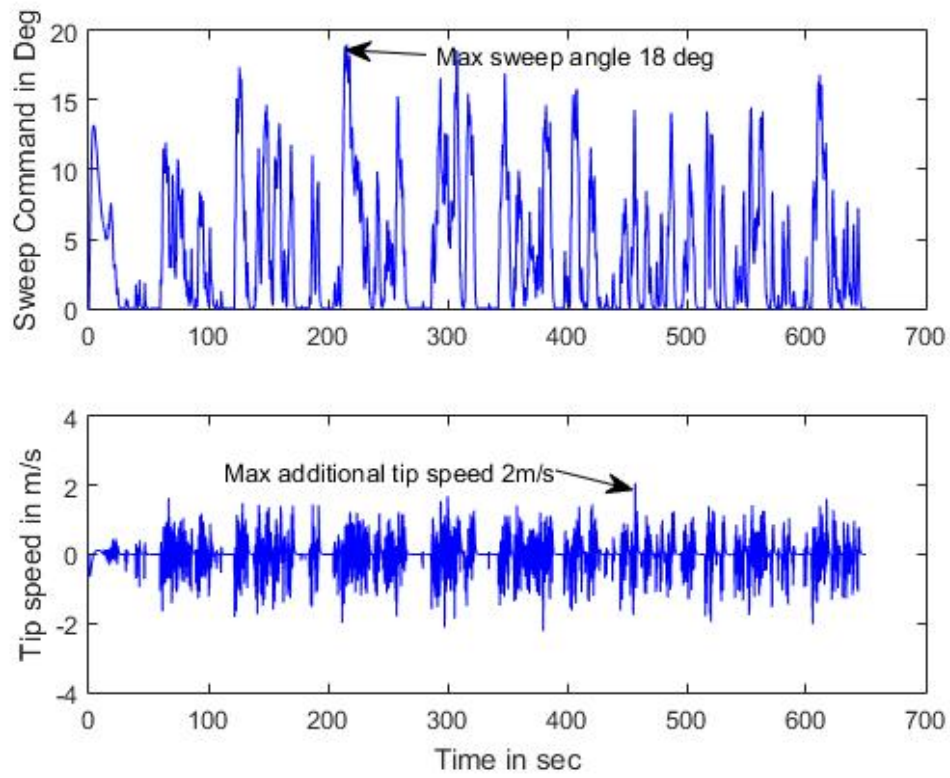

Fig. 17 Tip sweep angle response during a turbulent wind input for the modified NREL wind turbine.

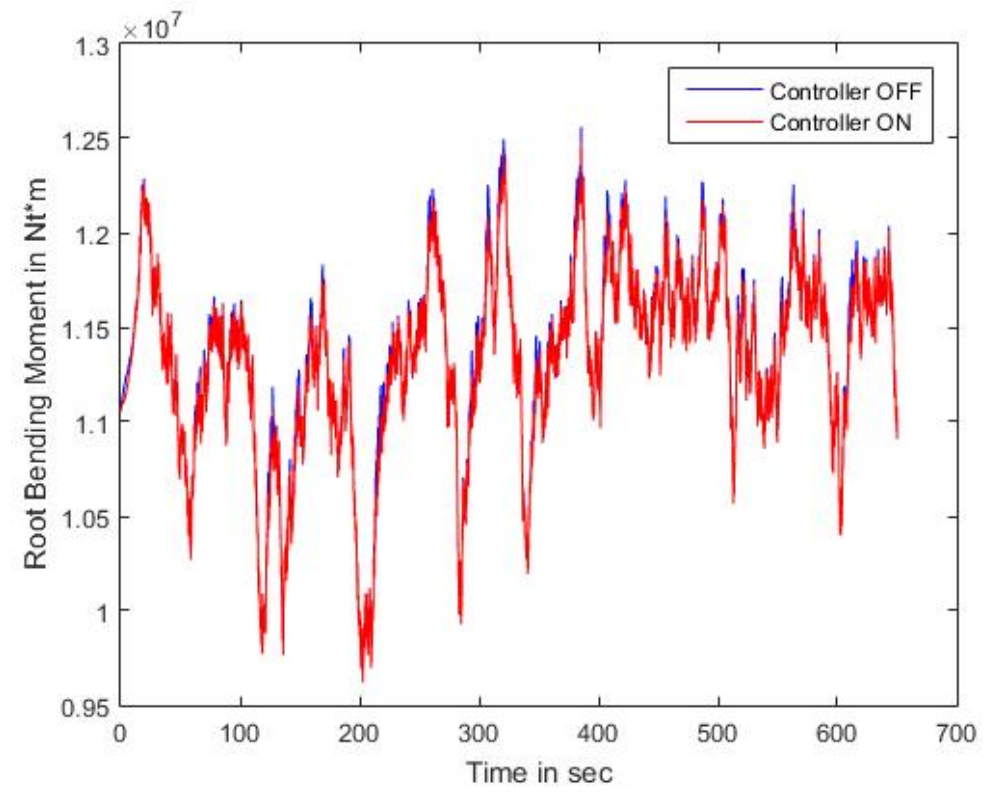

Fig. 18 Fatigue load reduction during a turbulent wind input for the modified NREL wind turbine.

The fatigue load reduction is estimated with the use of the MCrunch engineering tool, developed by NREL [21] MCrunch is a set of scripts initially developed for processing wind turbine test and simulation data, but it can be used for other applications, too. In this case, the blade root bending moment, is rainflow counted (using MCrunch) for the 600 sec simulation test of the modified wind turbine, with and without the controller operating at the 3-D turbulent wind field of figure 15. From the rainflow counting process the signal of the root bending moment is discretized into cycle amplitudes and the corresponding number of them. Then, the Damage Equivalent Load (DEL) is calculated for this two cases and the percent decrease in fatigue loading, yields.

In this work, the calculated DELs for the controller off and on cases, do not represent the actual equivalent loads, as the reference signal only contains the root bending moments. However, the calculated DEL number is directly connected to the damage equivalent load (as the stress is load divided by the cross 
section area which is not modified) and the corresponding percentage reduction is a reliable measure of the benefit.

Therefore, the calculated percentage fatigue load reduction is $\mathbf{3 . 2 \%}$ with the controller on and this is a noteworthy improvement considering the high loading operation of the wind turbine, at rated wind speed and turbulence intensity of $10 \%$. A full scale fatigue analysis for the entire envelope of the wind turbine would determine the total benefit.

\subsection{GUST LOAD REDUCTION}

One of the design requirements of a wind turbine according to [22] is tolerance to an extreme operating gust (EOG). EOG refers to the event of an abrupt rise in wind speed value, (not direction) that lasts for a few seconds. This consequently leads to peak loads that may compromise the integrity of the turbine's structure. The concept of a variable tip swept rotor could help alleviate those peak loads with a suitable control system that is capable of sweeping the blades in the case of an EOG event. So, in this paragraph the load reduction margin is calculated for the 5MW NREL wind turbine configured with a $10 \%$ swept rotor that is exposed to an EOG during its rated operation at $11.4 \mathrm{~m} / \mathrm{s}$ uniform wind speed and $12.1 \mathrm{rpm}$ rotation. This is a simplification of the International Electrotechnical Commission (IEC) case in terms of not including wind turbine fault, the rotor speed is constant and the rotor blades are stiff.

The gust is given by the following equation in accordance with paragraph 6.3.2.2 of [18]:

$$
V_{\text {gust }}=\operatorname{Min}\left\{1.35\left(V_{e 1}-V_{h u b}\right) ; 3.3\left(\frac{\sigma_{1}}{1+0,1\left(\frac{D}{\Lambda_{1}}\right)}\right)\right\}
$$

$\mathrm{V}_{\mathrm{e} 1}$ is $80 \%$ of the $\mathrm{V}_{50}$ extreme wind speed with a recurrence period of 50 years, $\sigma_{1}=0.11 \mathrm{~V}_{\text {hub }}, \Lambda_{1}$ is the turbulence scale parameter and $\mathrm{D}$ is the rotor diameter.

Then, equation (24) defines the wind speed variation in relation to time for a period of $\mathrm{T}=10.5 \mathrm{sec}$ which is depicted at Figure 19:

$$
V(z, t)=V(z)-0.37 V_{\text {gust }} \sin (3 \pi t / T)(1-\cos (2 \pi \tau / \mathrm{T})
$$

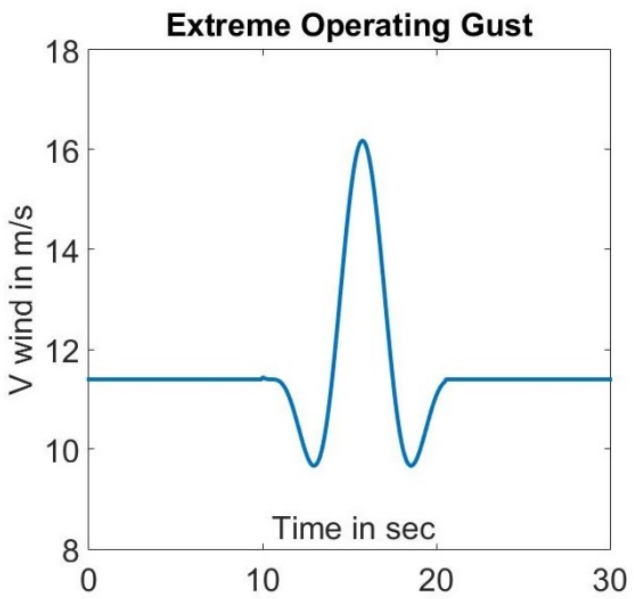

Fig. 19 An extreme operating gust according to IEC 61400-1 on the 5MW NREL wind turbine

Figure 20 shows in practice how a wind turbine with active tip sweep control capability can lower the blade loads during an extreme operating gust of Figure 19. The results derive from simulation runs for the $10 \%$ tip swept 5MW NREL (no blade extension) wind turbine that incorporates active sweep control using the BEM model. Specifically the controller doesn't react at the beginning of the gust $(t=50$ sec) when the velocity drops and the blade loads tend to drop accordingly. However, when the opposite phenomenon sets in it successively sweeps the rotor blade tips in order to lower the blade root bending moment. Then, the acceleration of wind velocity excites once again the controller but in a more gentle way as it recovers fast to the value of $11.4 \mathrm{~m} / \mathrm{s}$. The operating range of the controller for both cases is set to $0-25$ degrees and the 
gain and time constant of the high pass filter are found from the optimization process, explained above. It is noteworthy that load reduction is achieved through smooth and small deflections of the blade tips (in the order of $2 \mathrm{~m}$ ) which implies low inertia loads experienced by the control device.

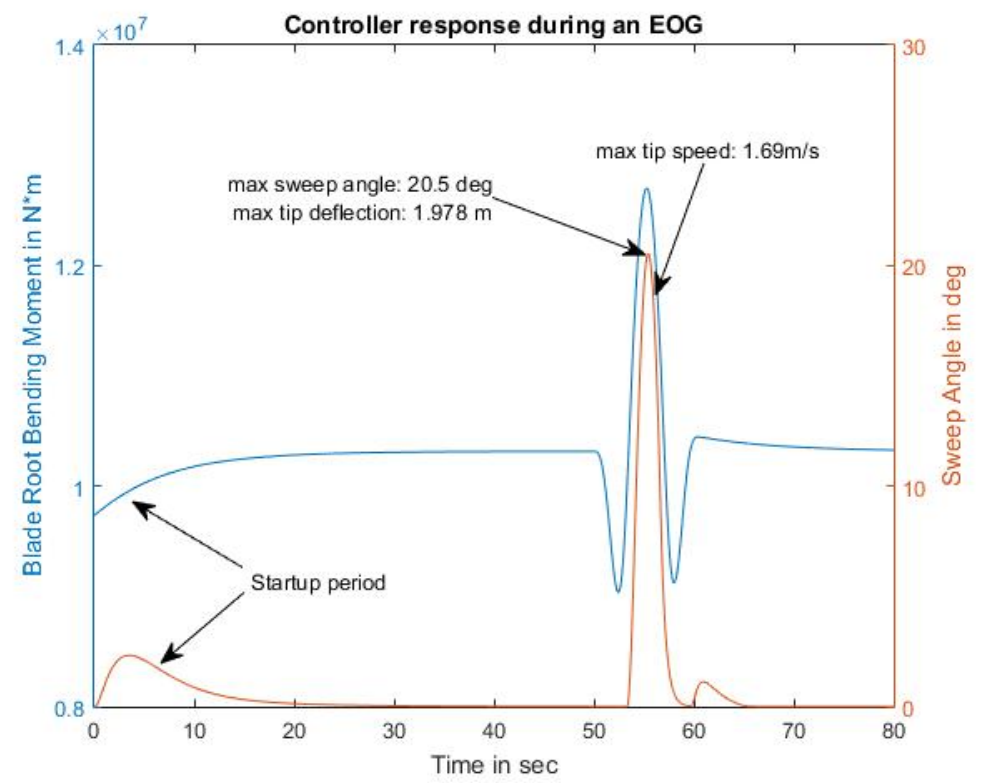

Fig. 20 Tip sweep angle response during an EOG for $10 \%$ tip swept rotor.

As it is seen in figure 21 (same simulation runs using BEM), the maximum load reduction for the $10 \%$ tip swept rotor is $2.63 \%$, which is an important result considering the small part of the blade that is swept. The capability of the controller in reducing the extreme loads could be taken into account in the design phase and lead to lighter blade structure. In addition, a reduction in the power peak, in the order of $1 \%$ as it is seen in figure 22, is observed which is beneficial in terms of introducing lower energy spikes into the grid.

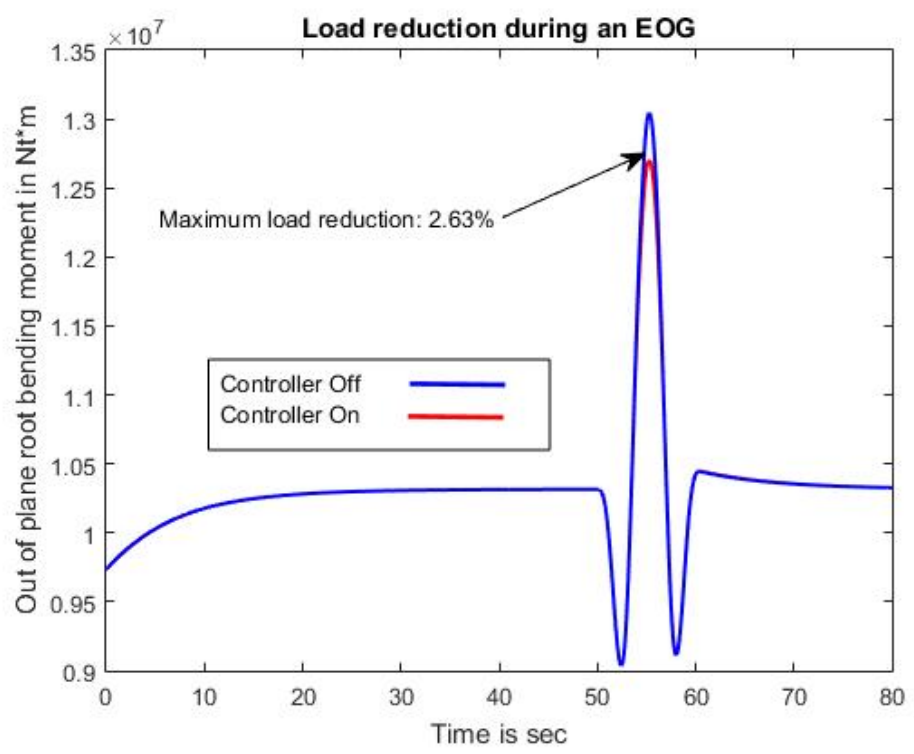

Fig. 21 Out of plane root bending moment of Blade No1 during an EOG for 10\% tip swept rotor. 
671

672

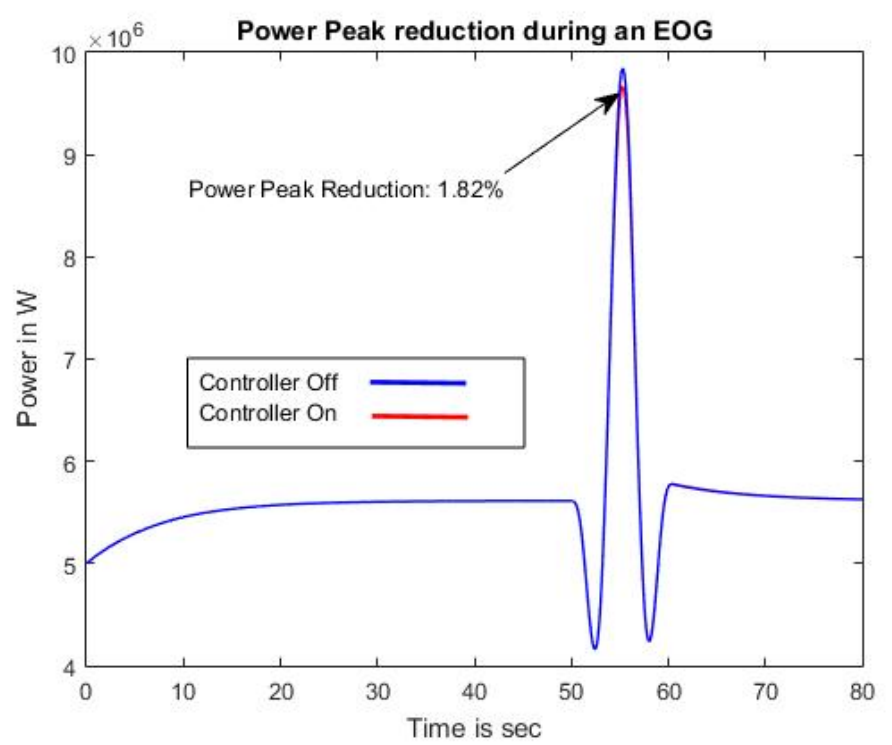

Fig. 22 Wind Turbine Power Peak reduction during an EOG for 10\% tip swept rotor.

After a small-scale study performed in [9] about the potential of this control scheme in reducing extreme loads, it is seen in figure 23 that even higher values in the order of $8 \%$ can be achieved for tip sweep percentage equal to $30 \%$.

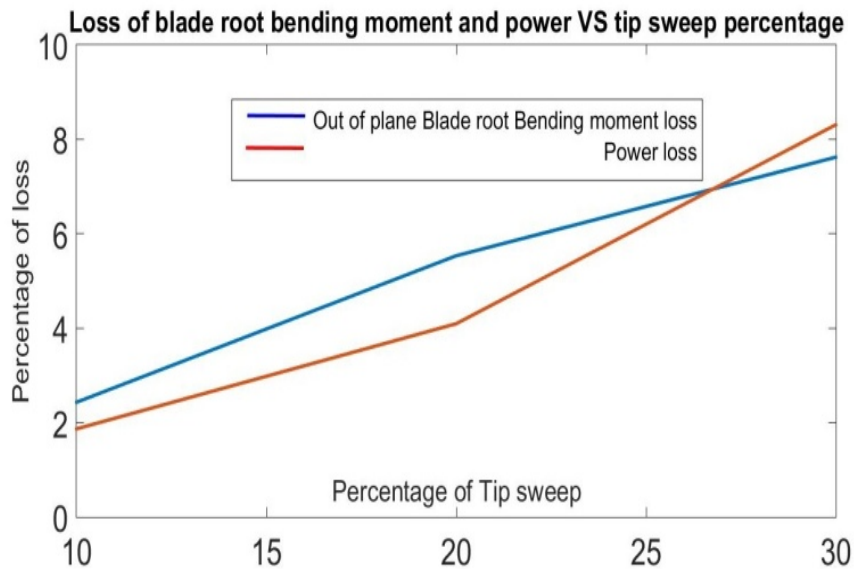

Fig. 23 Extreme load reduction and power peak reduction percentages in relation to rotor tip sweep percentage of 5MW NREL wind turbine.

\section{CONCLUSIONS}

In this work the variable tip sweep capability control concept is incorporated in the 5MW NREL reference wind turbine on a purely aerodynamic model. The originality that this research work incorporates is that the investigation is performed with a simple - engineering BEM model modified for tip swept rotors which is based on a more analytical model based on ULL theory. The early results from the parametric study with the ULL model that also agree with the results of the BEM model, indicate that there is a good potential in using active blade tip sweeping concept as a constant fatigue load alleviator or a safety feature during wind gusts. The improvement is noticeable in the 5MW reference wind turbine and could be more significant for larger wind turbines that experience higher tip speeds and deflections. However, power increase can only be achieved passively utilizing blade span extension combined with tip sweeping. In every case, there will be a trade off of benefits and drawbacks. For the fixed solution which is investigated 
first there will be an AEP increase with similar loads but this will add cost to the production phase expressed as extra development and constructions costs. Regarding the active solution, there will also be a gain in AEP accompanied with reduced fatigue / extreme loads but in this case the extra costs will be even more. As part of a future work there is a need to select the type of the actuator that will move the tip and design a suitable stable controller based on aeroelastic predictions, too. Of course this actuator will add weight to the structure and in addition would require the establishment of a maintenance plan which in turn raises the functional costs. The proposed solution is based on a simple controller and its performance could be improved with the design of an advanced controller that could provide more load regulation. The target of the proposed concept is to evaluate the benefits of an innovative conceptual active sweep controller and not to provide the optimal solution.

\section{ACKNOWLEDGEMENTS}

The research leading to these results has received funding from the People Programme (Marie Curie Actions) of the European Union's 7th Framework Programme FP7/2007-2013/ under REA grant agreement $\mathrm{n}^{\circ}$ [PCIG12-GA-2013-618756]

\section{REFERENCES}

[1] Barlas, T. K., and Van Kuik, G. A. M.. "Review of state of the art in smart rotor control research for wind turbines." Progress in Aerospace Sciences 46.1 (2010): 1-27.

[2] Riziotis, V. A., Manolas, D. I. and Voutsinas, S. G. "Free-wake Aeroelastic Modelling of Swept Rotor Blades.".EWEA, Brussels,2011.

[3] Maggio, T., Grasso F., and Coiro. D. P. "Numerical Study on Performance of Innovative Wind Turbine Blade for Load Reduction." EWEA, EWEC2011, Bruxelles (2011): 14-17.

[4] Ashwill, Thomas D. Sweep-twist adaptive rotor blade: final project report. No.SAND2009-8037. Sandia National Laboratories, 2010.

[5] Voutsinas, Spyros G. "Vortex methods in aeronautics: how to make things work." International Journal of Computational Fluid Dynamics 20.1 (2006): 3-18.

[6] Katz, Joseph, and Allen Plotkin. Low-speed aerodynamics. Vol. 13. Cambridge University Press, 2001

[7] Van Garrel, A. "Development of a wind turbine aerodynamics simulation module.", ECN Report ECN-C-03-079, 2003.

[8] Ansys, Inc Release 12.0, April 2009, www.ansys.com

[9] Boulamatsis A., Active Control of Wind Turbines Through Varying Blade Tip Sweep,PhD Thesis, 2017

[10] Butterfield, Sandy, Walter Musial, and George Scott. Definition of a 5-MW reference wind turbine for offshore system development. Golden, CO, Tech.Rep. NREL/TP-500-38060: National Renewable Energy Laboratory, 2009.

[11] Kloosterman, M. H. M. Development of the Near Wake behind a Horizontal Axis Wind Turbine Including the development of a Free Wake Lifting Line Code. Diss. M. Sc Dissertation, TUDelft, 2009.

[12] http://www.nvidia.com/object/cuda_home_new.html

[13] Fung, Yuan-cheng. An introduction to the theory of aeroelasticity. Courier Corporation, 2002.

[14] Pirrung, G. R., Morten Hartvig Hansen, and H. A. Madsen. "Improvement of a near wake model for trailing vorticity." Journal of Physics: Conference Series. Vol. 555. No. 1. IOP Publishing, 2014.

[15] Barlas, T. K. and van Kuik, G. A. M., Aeroelastic modelling and comparison of advanced active flap control concepts for load reduction on the Upwind 5MW wind turbine, Proceedings of the 2009 European Wind Energy Conference, Marseile, France, 2009.

[16] Øye, S. "Unsteady wake effects caused by pitch-angle changes." IEA R\&D WECS Joint Action on Aerodynamics of Wind Turbines, 1st Symposium. 1986.

[17] [4] Martin, O. L. "Hansen. Aerodynamics of wind turbines [M]. Earthscan published." Inc., New York (2008): 28-63.

[18] Snel, H., and J. G. Schepers. Joint investigation of dynamic inflow effects and implementation of an engineering method. Netherlands Energy Research Foundation ECN, 1995.

[19] Burton, Tony, et al. Wind energy handbook. John Wiley \& Sons, 2001

[20] Jonkman B.J., Kilche L., TurbSim User's Guide: Version 1.06.00, Technical Report NREL/TP, September 2012

[21] Marshall L. Buhl, Jr., Crunch User's guide, NREL, 2003.

[22] International Electrotechnical Commission IEC 61400-1 Ed.3, "Wind turbines - Part 1:Design Requirements", Tech Report 61400-1,IEC, 2005 


\section{Highlights}

- Active control of wind turbines through variable tip swept blades is a promising concept, mainly intended for fatigue and extreme blade load reduction.

- Power generation increase was not achieved through active control. It was achieved though, through longer tip swept rotor blades that induce a relatively low load penalty.

- A Blade Element Momentum theory based model combined with an innovative correction in the calculation of axial induction factor was developed in order to investigate the aerodynamics of variable tip swept rotors. 